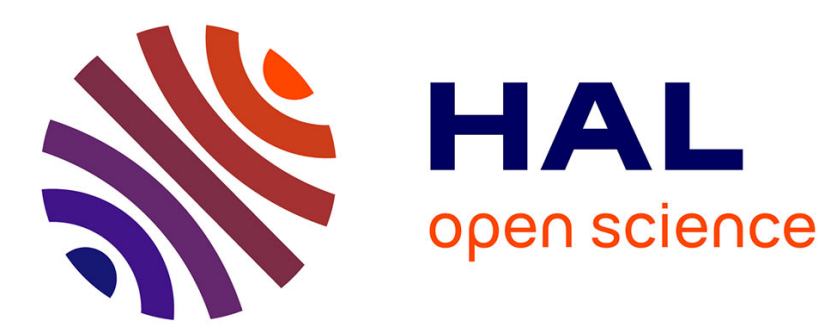

\title{
Using projection operators in computer aided geometric design
}

Laurent Busé, Mohamed Elkadi, Bernard Mourrain

\section{To cite this version:}

Laurent Busé, Mohamed Elkadi, Bernard Mourrain. Using projection operators in computer aided geometric design. Contemporary mathematics, 2003, 334, pp.321-342. inria-00098681

\section{HAL Id: inria-00098681 https://hal.inria.fr/inria-00098681}

Submitted on 25 Sep 2006

HAL is a multi-disciplinary open access archive for the deposit and dissemination of scientific research documents, whether they are published or not. The documents may come from teaching and research institutions in France or abroad, or from public or private research centers.
L'archive ouverte pluridisciplinaire HAL, est destinée au dépôt et à la diffusion de documents scientifiques de niveau recherche, publiés ou non, émanant des établissements d'enseignement et de recherche français ou étrangers, des laboratoires publics ou privés. 


\title{
Using projection operators in Computer Aided Geometric Design
}

\author{
Laurent Busé, Mohamed Elkadi, and Bernard Mourrain
}

\begin{abstract}
We give an overview of resultant theory and some of its applications in computer aided geometric design. First, we mention different formulations of resultants, including the projective resultant, the toric resultant, and the residual resultants. In the second part we illustrate these tools, and others projection operators, on typical problems as surface implicitization, inversion, intersection, and detection of singularities of a parameterized surface.
\end{abstract}

\section{Introduction}

The aim of this paper is to present a general overview of projection operators used in EAG (Effective Algebraic Geometry) and their implication in CAGD (Computer Aided Geometric Design). A projection operator is an operator which associates to an overdetermined polynomial system in several variables a polynomial depending only on the coefficients of this system, which vanishes when the system has a solution. This projection operation is a basic ingredient of many methods in EAG. In this paper, we describe a general framework, based on our recent works on resultants, in order to handle the known resultant formulations (projective, toric, residual) in a uniform way. These constructions are special cases of the projection of the incidence variety associated with line bundles, very ample almost everywhere on a given projective variety. This will allow us to handle the critical problems of base points, occurring in many situations. Special applications to the problem of implicitization in CAGD are given. In particular, we describe how the different resultant constructions apply to this problem and propose a new method based on approximation complexes, extending the method of moving surface, and which allows us to treat general base points. These constructions are illustrated on 3 typical problems occurring in CAGD, namely surface inversion, intersection, and detection of singularities of a parameterized surface. We point out that this approach based on resultant constructions yields a preprocessing step in which we generate a dedicated code for the problem we want to handle. The effective resolution, which then requires the instantiation of the parameters of problems and the numerical solving, could thus be highly accelerated. Experimental details, which would lead us outside the scope of this paper, are not given, but examples based on the maple package multires illustrate our presentation.

The paper is organized as follows: In section 2 we give a general construction of the resultant theory. In section 3 we obtain as particular cases of the previous construction a several usual resultants (classical, anisotropic, toric, residual) and 
we show how to compute them. In section 4 we focus on the implicitization problem for rational parametric $3 D$-surfaces using different resultant formulations, moving quadrics, approximation complexes, Bezoutians, .... In section 5 by means of resultant techniques we study some problems in CAGD: surface inversion (i.e. find the inverse images of points in a parametric rational surface), intersection (i.e. intersect a parametric curve and an implicit surface), detection of singularities of an implicit surface, ... We will see that these questions reduce to linear algebra by the use of elimination theory.

Hereafter $\mathbb{K}$ is an algebraically closed field.

\section{Resultant theory}

The theory of resultant is devoted to the study of conditions on the coefficients of an overdetermined system to have a solution in a fixed variety. The most popular resultant is the so-called Sylvester's resultant of two univariate polynomials $f_{0}(x)=c_{0,0}+c_{0,1} x+\ldots+c_{0, n} x^{n}$ and $f_{1}(x)=c_{1,0}+c_{1,1} x+\ldots+c_{1, m} x^{m}$. It is an irreducible polynomial in the coefficient ring $\mathbb{K}\left[c_{0,0}, \ldots, c_{0, n}, c_{1,0}, \ldots, c_{1, m}\right]$, which is usually denoted $\operatorname{Res}\left(f_{0}, f_{1}\right)$. For a given specialization of these coefficients, $\operatorname{Res}\left(f_{0}, f_{1}\right)$ vanishes if and only if one of the following conditions is satisfied:

- $f_{0}$ and $f_{1}$ have a common root in $\mathbb{K}$

- $\operatorname{deg}(f)<n$ and $\operatorname{deg}(g)<m$, i.e. $c_{0, n}=c_{1, m}=0$

is satisfied. These two conditions can be replaced by the single one: $f_{0}$ and $f_{1}$ have a common root in the projective space $\mathbb{P}_{\mathbb{K}}^{1}$. It appears that the projective setting is more simple here, and this is also true in a more general situation. Consequently, in what follows, we will always work on projective varieties.

The typical situation is the case of a system of $n+1$ equations in a projective variety $X$ of dimension $n$, of the form:

$$
\mathbf{f}_{\mathbf{c}}:=\left\{\begin{aligned}
f_{0}(x) & =\sum_{j=0}^{k_{0}} c_{0, j} \psi_{0, j}(x) \\
\vdots & \\
f_{n}(x) & =\sum_{j=0}^{k_{n}} c_{n, j} \psi_{n, j}(x)
\end{aligned}\right.
$$

where $\mathbf{c}=\left(c_{i, j}\right)$ are parameters, $x$ is a point of $X$, and such that for all $i=0, \ldots, n$ we have a regular map (independent of $\mathbf{c}$ )

$$
\phi_{i}: x \in X \mapsto\left(\psi_{i, 0}(x): \ldots: \psi_{i, k_{i}}(x)\right) \in \mathbb{P}^{k_{i}} .
$$

In the language of modern algebraic geometry, to each map $\phi_{i}$ is associated an invertible sheaf $\mathcal{L}_{i}=\phi^{*}\left(\mathcal{O}_{\mathbb{P} k_{i}}(1)\right)$, and a vector subspace $V_{i}=\left\langle\psi_{i, 0}, \ldots, \psi_{i, k_{i}}\right\rangle$ of its global sections $\Gamma\left(X, \mathcal{L}_{i}\right)$ (see $[\mathbf{H a r} 77]$, II.7). In this way, the $\mathbb{K}$-vector space $V_{i}$ parameterizes all the polynomials $f_{i}$ that we can obtain by specializing the coefficients $\left(c_{i, j}\right)_{j=0, \ldots, k_{i}}$ in $\mathbb{K}$. As two polynomials $f_{i}$ and $g_{i}$ such that $f_{i}=\lambda g_{i}$ with $\lambda \in \mathbb{K}^{*}$ define the same zero locus, it is convenient to identify them, and hence to parameterize polynomials $f_{i}$ by the projective space $\mathbb{P}\left(V_{i}\right) \simeq \mathbb{P}^{k_{i}}$.

The projection (or elimination) problem consists, in this case, in finding necessary (and sufficient) conditions on $\mathbf{c}$ such that the system $\mathbf{f}_{\mathbf{c}}=0$ has a solution in $X$. Considering a geometric point of view, we look for the values of parameters $\mathbf{c}=\left(c_{i, j}\right) \in \mathbb{P}^{k_{0}} \times \cdots \times \mathbb{P}^{k_{n}}$ such that there exists $x \in X$ with $f_{i}(x)=\sum_{j=0}^{k_{i}} c_{i, j} \psi_{i, j}(x)=0$ for $i=0, \ldots, n$. In other words, $\mathbf{c}$ is the first projection of the point $(\mathbf{c}, x)$ in the incidence variety

$$
W_{X}=\left\{(\mathbf{c}, x) \in \mathbb{P}^{k_{0}} \times \cdots \times \mathbb{P}^{k_{n}} \times X: f_{i}(x)=0, i=0, \ldots, n\right\} .
$$


We denote by $\pi_{1}: W_{X} \rightarrow \mathbb{P}^{k_{0}} \times \cdots \times \mathbb{P}^{k_{n}}$ and $\pi_{2}: W_{X} \rightarrow X$ the first and second projections. The image by $\pi_{2}$ of a point of $W_{X}$ is a solution in $X$ of the associated system, and the image of $W_{X}$ by $\pi_{1}$ is precisely the set of values of parameters $\mathbf{c}$ for which the system has a root in $X$. We define the resultant of $f_{0}, \ldots, f_{n}$ when $\pi_{1}\left(W_{X}\right)$ is an irreducible hypersurface, and we denote $\operatorname{Res}_{V_{0}, \ldots, V_{n}}$ its equation (unique up to a non-zero multiple in $\mathbb{K}$ ).

Definition 2.1. Let $\mathcal{L}$ be an invertible sheaf on $X$ and $V$ be a vector subspace of the vector space of its global sections $H^{0}(X, \mathcal{L})$.

- The base points of $V$ are the points $x \in X$ such that $f(x)=0$ for all $f \in V$.

- $V$ is said to be very ample if the canonical map

$$
x \in X \mapsto\{f \in V: f(x)=0\} \in \mathbb{P}(V)
$$

is an embedding, or equivalently, if $V$ separates the points and the tangent vectors in $X$ (see [GH78] p.180).

- $V$ is said to be very ample almost everywhere if there exists a dense open subset $U$ of $X$ such that the restricted map

$$
x \in U \mapsto\{f \in V: f(x)=0\} \in \mathbb{P}(V)
$$

is an embedding, or equivalently, if $V$ separates the points and the tangent vectors in $U$.

THEOREM 2.2. ([BEM01] proposition 1) Suppose that each $V_{i}$ is very ample almost everywhere and has no base points, then $\pi_{1}\left(W_{X}\right)$ is a hypersurface of $\prod_{i=0}^{n} \mathbb{P}^{k_{i}}$. Its degree in the coefficients of $f_{i}$ (that is w.r.t. to $\mathbb{P}^{k_{i}}$ ) is $\int_{X} \prod_{j \neq i} c_{1}\left(\mathcal{L}_{j}\right)$, where $c_{1}\left(\mathcal{L}_{j}\right)$ denotes the first Chern class of the invertible sheaf $\mathcal{L}_{j}$.

REMARK 2.3. It is clear that if $V_{i}$ is very ample then $V_{i}$ has no base points and $V_{i}$ is very ample almost everywhere. Consequently the mixed resultant of [GKZ94] is contained in this theorem.

If the system $\mathbf{f}_{\mathbf{c}}$ satisfies the hypothesis of theorem $2.2, \operatorname{Res}_{V_{0}, \ldots, V_{n}}$ is a function on $\prod_{i=0}^{n} \mathbb{P}^{k_{i}}$ satisfying the property

$$
\operatorname{Res}_{V_{0}, \ldots, V_{n}}\left(f_{0}, \ldots, f_{n}\right)=0 \Leftrightarrow \exists x \in X: f_{0}(x)=\cdots=f_{n}(x)=0 .
$$

By construction $\operatorname{Res}_{V_{0}, \ldots, V_{n}}$ is multihomogeneous, its degree in the coefficients of $f_{i}$ is given by the "explicit formula" $\int_{X} \prod_{j \neq i} c_{1}\left(\mathcal{L}_{j}\right)$. This number can be seen as the number of solutions of a generic system $\left\{x \in X: f_{j}(x)=0: j=0, \ldots, n, j \neq i\right\}$.

As we will see in the next section, a lot of known resultants as classical resultants, toric resultants or anisotropic resultants are obtained from theorem 2.2 by choosing $X$ and $V_{0}, \ldots, V_{n}$ adequately. However this construction of resultant degenerates if the system $\mathbf{f}_{\mathbf{c}}$ has base points (i.e. $\pi_{1}\left(W_{X}\right)=\prod_{i=0}^{n} \mathbb{P}^{k_{i}}$ ). Such systems with base points arise very often in practice, so we now generalize the preceding construction of resultants, taking into account the possible presence of base points.

From now on, we only suppose that the maps $\phi_{i}$ are rational and not regular (i.e. possibly with base points), each vector space $V_{i}$ being a subvector space of the global sections of a given invertible sheaf $\mathcal{L}_{i}$. We will use a standard tool in algebraic geometry to "erase" base points, called the blowing-up. The basic idea is to blow-up $X$ along the base points locus of the system $\mathbf{f}_{\mathbf{c}}$, then obtain a new projective variety $\tilde{X}$ of the same dimension where the pull-back of our system $\mathbf{f}_{\mathbf{c}}$ 
can be seen without base points, and finally apply theorem 2.2 . Roughly speaking we blow-up the ideal of $X$ associated to the union of base points of each $V_{i}$, for $i=0, \ldots, n$. More precisely, we blow-up the ideal sheaf $\mathcal{I}$ on $X$ obtained as the image of the morphism of sheaves

$$
\left(\oplus_{i=0}^{n} V_{i}\right) \otimes_{\mathbb{K}}\left(\oplus_{i=0}^{n} \mathcal{L}_{i}^{*}\right) \rightarrow \mathcal{O}_{X},
$$

induced by the canonical morphism

$$
\oplus_{i=0}^{n} V_{i} \otimes_{\mathbb{K}} \mathcal{O}_{X} \rightarrow \oplus_{i=0}^{n} \mathcal{L}_{i} .
$$

We denote the blow-up of $X$ along $\mathcal{I}$ by $\pi: \tilde{X} \rightarrow X$. We have the new incidence variety

$$
W_{\tilde{X}}=\left\{(\mathbf{c}, x) \in \mathbb{P}^{k_{0}} \times \cdots \times \mathbb{P}^{k_{n}} \times \tilde{X}: \tilde{f}_{i}(x)=0, i=0, \ldots, n\right\},
$$

where $\tilde{f}_{i}$ denotes the virtual transform of $f_{i}$ by $\pi$, that is the pull-back $\pi^{*}\left(f_{i}\right)$ of $f_{i}$ seen as a section of $\pi^{*}\left(\mathcal{L}_{i}\right) \otimes \pi^{-1} \mathcal{I}$. $\mathcal{O}_{\tilde{X}}$. Denoting by $\tilde{\pi}_{1}: W_{\tilde{X}} \rightarrow \mathbb{P}^{k_{0}} \times \cdots \times \mathbb{P}^{k_{n}}$ and $\pi_{2}: W_{\tilde{X}} \rightarrow \tilde{X}$ the two natural projections, we obtain the following corollary of theorem 2.2 .

Corollary 2.4. ([Bus01a] proposition 2.2.4) Suppose that each $V_{i}$ is very ample almost everywhere, then $\tilde{\pi}_{1}\left(W_{\tilde{X}}\right)$ is a hypersurface of $\prod_{i=0}^{n} \mathbb{P}^{k_{i}}$. Its degree in the coefficients of $f_{i}$ (that is w.r.t. to $\mathbb{P}^{k_{i}}$ ) is given by $\int_{X} \prod_{j \neq i} c_{1}\left(\mathcal{L}_{j}\right) \otimes \pi^{-1} \mathcal{I}$. $\mathcal{O}_{\tilde{X}}$.

Moreover if there is no base points, then the ideal sheaf $\mathcal{I}$ is exactly $\mathcal{O}_{X}$, and $\pi$ is the identity $X \rightarrow X$ so that we recover the construction of resultants of theorem 2.2. Consequently, as soon as the $V_{i}$ 's are very ample almost everywhere, we construct a resultant for the system $\mathbf{f}_{\mathbf{c}}$ denoted by $\operatorname{Res}_{V_{0}, \ldots, V_{n}}$ and defined as the equation of the hypersurface $\tilde{\pi}_{1}\left(W_{\tilde{X}}\right)$. It is, as usual, multihomogeneous and satisfies

$$
\operatorname{Res}_{V_{0}, \ldots, V_{n}}\left(f_{0}, \ldots, f_{n}\right)=0 \Leftrightarrow \exists x \in \tilde{X}: \tilde{f}_{0}(x)=\cdots=\tilde{f}_{n}(x)=0 .
$$

Notice that this resultant depend only on the birational equivalent class of $X$ and the vector spaces $V_{0}, \ldots, V_{n}$ (and not on the $\mathcal{L}_{i}$ 's; see [Bus01a] chapter 2 for more details).

We have thus constructed a general resultant which is valid for a very large range of systems $\mathbf{f}_{\mathbf{c}}$, but it remains to compute it!

\section{Examples of resultant constructions}

In this section we give several examples of resultants as particular cases of the previous construction and show how to compute them.

The resultant is basically an elimination operator. It can be computed (at least theoretically) using Gröbner bases methods. However such methods are not used in practice for at least two reasons: because of the complexity issues and the high cost of Gröbner bases, and especially because the output is the expanded resultant itself. The methods we are going to present here give the resultant in a matrix formulation, which is much more adapted to applications. There is basically three ways to obtain such a formulation:

- As gcd of maximal minors of a surjective matrix.

- As a determinant of a complex (see [GKZ94], appendix A).

- As a ratio $\frac{\operatorname{det}(M)}{\operatorname{det}(E)}$ of two determinants, where $E$ is a submatrix of $M$. 
Notice that the two first points are always going together, coming from the knowledge of a complex which "resolves" the generic system of the corresponding resultant.

3.1. Classical resultant. The classical case studied in [Mac02], [vdW50], is the case where $X$ is the projective space $\mathbb{P}^{n}$ and $V_{i}$, for $i=0, \ldots, n$, is the vector of all monomials of a fixed degree $d_{i}$. Clearly, when $d_{i} \geq 1$ each $\mathcal{L}_{i}=\mathcal{O}_{X}\left(d_{i}\right)$ separates the points and the tangent vectors and thus $\operatorname{Res}_{V_{0}, \ldots, V_{n}}$ is well defined. It is traditionally denoted $\operatorname{Res}_{\mathbb{P} n}$. By theorem 2.2 (or Bézout theorem), its degree with respect to $V_{i}$ is $\prod_{j \neq i} d_{j}$.

The necessary and sufficient condition on $\mathbf{c}$ such that $f_{0}, \ldots, f_{n}$ have a common root in $\mathbb{P}^{n}$ is $\operatorname{Res}_{\mathbb{P}^{n}}\left(\mathbf{f}_{\mathbf{c}}\right)=0$. Macaulay's construction [Mac02] of the classical resultant can be seen as an extension of Sylvester's method to the multivariate case. We describe it in the affine setting by substituting $x_{0}=1, x_{1}=t_{1}, \ldots, x_{n}=t_{n}$.

Let $\nu=\sum_{i=0}^{n} d_{i}-n$ and $\mathbf{t}^{F}$ be the set of all monomials in $\mathbf{t}$ of degree $\leq \nu$. It contains $\left(\begin{array}{c}\nu+n \\ n\end{array}\right)$ elements. Let $t_{n}^{d_{n}} \mathbf{t}^{E_{n}}$ be the set of all monomials of $\mathbf{t}^{F}$ which are divisible by $t_{n}^{d_{n}}$. For $i=n-1, \ldots, 1$, we define by induction $t_{i}^{d_{i}} \mathbf{t}^{E_{i}}$ to be the set of all monomials of $\mathbf{t}^{F} \backslash\left(t_{n}^{d_{n}} \mathbf{t}^{E_{n}} \cup \ldots \cup t_{i+1}^{d_{i+1}} \mathbf{t}^{E_{i+1}}\right)$ which are divisible by $t_{i}^{d_{i}}$. The set $\mathbf{t}^{F} \backslash\left(t_{n}^{d_{n}} \mathbf{t}^{E_{n}} \cup \ldots \cup t_{1}^{d_{1}} \mathbf{t}^{E_{1}}\right)$ is denoted by $\mathbf{t}^{E_{0}}$ and is equal to

$$
\mathbf{t}^{E_{0}}=\left\{t_{1}^{\alpha_{1}} \cdots t_{n}^{\alpha_{n}}: 0 \leq \alpha_{i} \leq d_{i}-1\right\} .
$$

It has $d_{1} \cdots d_{n}$ monomials. $\mathbf{t}^{E}$

If $E$ is a subset of $\mathbb{N}^{n},\left\langle\mathbf{t}^{E_{n}}\right\rangle$ denotes the vector subspace generated by the set

The resultant matrix $\mathrm{S}$ is the matrix in monomial bases of the following linear map:

$$
\begin{aligned}
\mathcal{S}:\left\langle\mathbf{t}^{E_{0}}\right\rangle \times \cdots \times\left\langle\mathbf{t}^{E_{n}}\right\rangle & \rightarrow\left\langle\mathbf{t}^{F}\right\rangle \\
\left(q_{0}, \ldots, q_{n}\right) & \mapsto \sum_{i=0}^{n} q_{i} f_{i} .
\end{aligned}
$$

The determinant of $\mathrm{S}$ is generically not 0 (for it does not vanish when we specialize $f_{i}$ to $t_{i}^{d_{i}}$ ) and has the same degree $\prod_{i=1}^{n} d_{i}$ as the resultant with respect to $V_{0}$. Therefore

$$
\operatorname{det}(\mathbf{S})=\operatorname{Res}_{\mathbb{P} n}\left(\mathbf{f}_{\mathbf{c}}\right) \Delta\left(f_{1}, \ldots, f_{n}\right),
$$

where $\Delta\left(f_{1}, \ldots, f_{n}\right)$ is a subminor of $\mathbf{S}$ depending only on the coefficients of $f_{1}, \ldots, f_{n}[$ Mac02].

We remark that, if $R=\mathbb{K}\left[t_{1}, \ldots, t_{n}\right]$, the map (1) is in fact connected to the first map of the Koszul complex of the sequence $f_{0}, \ldots, f_{n}$,

$$
0 \rightarrow \wedge^{n} R^{n} \stackrel{d_{n}}{\longrightarrow} \wedge^{n-1} R^{n} \longrightarrow \cdots \longrightarrow R^{n} \stackrel{d_{1}}{\longrightarrow} R
$$

in degree $\nu$, where $d_{l}\left(e_{i_{1}} \wedge \cdots \wedge e_{i_{l}}\right)=\sum_{j=1}^{l}(-1)^{j} f_{i_{j}} e_{i_{1}} \wedge \cdots \widehat{e_{i_{1}}} \cdots \wedge e_{i_{l}}$. Indeed as shown in [Dem84], [Cha93] the determinant of the Koszul complex is the classical resultant of $f_{0}, \ldots, f_{n}$. For other constructions, also related to the Koszul complex and its dual which also yield the classical resultant sometimes in a more compact way, we refer to [Jou97], [WZ94], [DD01].

This resultant has been widely studied, and has a lot of properties; a quasicomplete list can be found in [Jou91]. We recall two of them that we will use later, a weight invariance property and the so-called Poisson's formula. 
For $i=0 \ldots n$, let $f_{i}=\sum_{|\alpha|=d_{i}} c_{\alpha, i} \mathbf{x}^{\alpha}$ be the generic homogeneous polynomial of degree $d_{i}$. The coefficients $c_{\alpha, i}$ are considered as indeterminates, that is $f_{i} \in A[\mathbf{x}]$ where $A$ denotes the coefficient ring $\mathbb{Z}\left[c_{\alpha, i},|\alpha|=d_{i}\right]$.

Lemma 3.1. ([Jou91] 5.13.2) Let $m$ be a fixed integer in $\{0,1, \ldots, n\}$. We graduate the coefficient ring $A$ by setting $\operatorname{deg}\left(c_{\alpha, i}\right)=\alpha_{m}$. Then $\operatorname{Res}_{\mathbb{P}^{n}}\left(f_{0}, \ldots, f_{n}\right) \in$ $\mathbb{Z}\left[c_{\alpha, i},|\alpha|=d_{i}\right]$ is isobar (i.e. homogeneous for this graduation) of weight $\prod_{i=0}^{n} d_{i}$ in $A$.

This lemma is a corollary of a more general formula called the "changing basis formula" (see [Jou91] 5.12). We end this section with the well-known Poisson's formula. For all $i=0, \ldots, n$, let $\tilde{f}_{i}\left(x_{1}, \ldots, x_{n}\right):=f_{i}\left(1, x_{1}, \ldots, x_{n}\right)$ and $\bar{f}_{i}\left(x_{0}, \ldots, x_{n-1}\right):=f_{i}\left(0, x_{1}, \ldots, x_{n}\right)$.

Lemma 3.2. ([Jou91] 2.7, [CLO97] III.3.5) Let $\rho=\operatorname{Res}_{\mathbb{P}^{n-1}}\left(\bar{f}_{1}, \ldots, \bar{f}_{n}\right) \in A$. We have

$$
\operatorname{Res}_{\mathbb{P}^{n}}\left(f_{0}, \ldots, f_{n}\right)=\operatorname{det}\left(\mathrm{M}\left(\tilde{f}_{0}\right)\right) \operatorname{Res}_{\mathbb{P}^{n-1}}\left(\bar{f}_{1}, \ldots, \bar{f}_{n}\right)^{d_{0}},
$$
where $\mathrm{M}\left(\tilde{f}_{0}\right)$ is the multiplication by $\tilde{f}_{0}$ in $A_{\rho}\left[x_{1}, \ldots, x_{n}\right] /\left(\tilde{f}_{1}, \ldots, \tilde{f}_{n-1}\right)$.

3.2. Anisotropic resultant. This resultant was introduced and studied by Jouanolou in [Jou91] and [Jou96]. It is a generalization of the classical resultant, taking into account the possible combinatorial properties of a polynomial system and giving a more "reduced" eliminant polynomial. Instead of considering all the variables $x_{0}, \ldots, x_{n}$ of the same degree 1 , we consider them with different weights.

Let $m_{0}, m_{1}, \ldots, m_{n}$ in $\mathbb{N}^{*}$. Set $\mu=\operatorname{lcm}\left(m_{0}, \ldots, m_{n}\right), \delta=\operatorname{gcd}\left(m_{0}, \ldots, m_{n}\right)$, and $\Delta=\frac{m_{0} m_{1} \ldots m_{n}}{\delta} \in \mathbb{N}$. We denote by $C$ the polynomial ring $\mathbb{K}\left[x_{0}, \ldots, x_{n}\right]$ with $\operatorname{deg}\left(x_{i}\right)=1$, and by ${ }^{a} C$ the same polynomial ring but with $\operatorname{deg}\left(x_{i}\right)=m_{i}$ (the exponent $a$ stands for anisotropic). Usually we consider the projective space $\mathbb{P}^{n}=\operatorname{Proj}(C)$, but here we work on ${ }^{a} \mathbb{P}^{n}=\operatorname{Proj}\left({ }^{a} C\right)$, that is the anisotropic projective space with weights $\left(m_{0}, \ldots, m_{n}\right)$. Notice that from a geometrical point of view we have the canonical morphism

$$
\left(x_{0}: \cdots: x_{n}\right) \in \mathbb{P}^{n} \mapsto\left(x_{0}^{m_{0}}: \cdots: x_{n}^{m_{n}}\right) \in{ }^{a} \mathbb{P}^{n} .
$$

Let $X={ }^{a} \mathbb{P}^{n}$ and $V_{i}$, for all $i=0, \ldots, n$, be the set of all isobar (i.e. homogeneous in the weighted variables) monomials of degree $d_{i}$ in ${ }^{a} C$, that is $V_{i}$ is the vector space of global sections of the invertible sheaf $\mathcal{L}_{i}=\mathcal{O}_{a \mathbb{P}_{\mathbb{K}}^{n}}\left(d_{i}\right)$. In section 3.1, we required that $d_{i} \geq 1$ to fulfill the very ampleness condition for the existence of the resultant. Here we have a similar hypothesis by assuming that $\mu \mid d_{i}$ for all $i=0, \ldots, n$. In this way the resultant $\operatorname{Res}_{V_{0}, \ldots, V_{n}}$, denoted ${ }^{a} \operatorname{Res} \mathbb{P}^{n}$, is well defined. It is also multi-homogeneous, and its degree with respect to the coefficients of the polynomial $f_{i}$ is $\frac{\prod_{j \neq i} d_{j}}{\Delta}$ (see [Jou91] 6.3.5(A)).

As for the classical resultant, there are different ways to compute it, the more commonly one is the anisotropic Macaulay's matrices, coming from the anisotropic Koszul complex (see [Jou96]). Anisotropic resultant and classical resultant are closely related, and almost all the classical resultant properties (as Poisson's formula) can be extended to the anisotropic situation. To illustrate it, we give the following result which shows how the anisotropic situation reduces to the classical one.

LEMma 3.3. ([Jou91] 6.3.5(B)) Let $f_{0}, \ldots, f_{n}$ be isobar polynomials in ${ }^{a} C$ of respective degree $d_{i}$, and let $f_{i}^{\sharp}\left(x_{0}, \ldots, x_{n}\right)=f_{i}\left(x_{0}^{m_{0}}, \ldots, x_{n}^{m_{n}}\right) \in C$. We have

$$
\operatorname{Res}_{\mathbb{P} n}\left(f_{0}^{\sharp}, \ldots, f_{n}^{\sharp}\right)={ }^{a} \operatorname{Res} \mathbb{P}^{n}\left(f_{0}, \ldots, f_{n}\right)^{\Delta} .
$$


3.3. Toric resultant. The toric (or sparse) resultant has been introduced in [KSZ92], then developed in [GKZ94]. It takes into account the monomial support of the input polynomials. Thus it is possible to work with polynomials having negative exponents, that is Laurent polynomials. Let $f_{i}(\mathbf{t})=\sum_{\alpha \in A_{i}} \mathbf{c}_{\alpha, i} \mathbf{t}^{\alpha}, i=$ $0 \ldots n$, be $n+1$ Laurent polynomials (where $\mathbf{t}=\left(t_{1}, \ldots, t_{n}\right)$ ) with supports into fixed sets $A_{i} \subset \mathbb{Z}^{n}$. To each finite set $A_{i} \subset \mathbb{Z}^{n}$ we can associate a projective toric variety $X_{A_{i}}$ (not necessary normal, see [GKZ94] chapter 5) which can be defined as the algebraic closure of the image of the map

$$
\sigma_{i}:\left(\mathbb{K}^{*}\right)^{n} \rightarrow \mathbb{P}^{N_{i}}: \mathbf{t} \mapsto\left(\mathbf{t}^{\alpha}\right)_{\alpha \in A_{i}}
$$

where $N_{i}=\left|A_{i}\right|-1$. Each $f_{i}(\mathbf{t})$ can thus be extended globally (by "homogenization") as a linear form on $X_{A_{i}}$. In order to apply the previous resultant theory, we consider the projective variety $X$ obtained as the algebraic closure of the image of the map

$$
\begin{aligned}
\sigma:\left(\mathbb{K}^{*}\right)^{n} & \rightarrow X_{A_{0}} \times \ldots \times X_{A_{n}} \\
\mathbf{t} & \mapsto\left(\mathbf{t}^{\alpha}\right)_{\alpha \in A_{0}} \times \ldots \times\left(\mathbf{t}^{\alpha}\right)_{\alpha \in A_{n}} .
\end{aligned}
$$

Denoting by $\mathbb{K}^{A_{i}}$ the subspace of polynomials with support in $A_{i}$, by construction, $X \subset X_{A_{0}} \times \ldots \times X_{A_{n}} \subset \mathbb{P}\left(\mathbb{K}^{A_{0}{ }^{*}}\right) \times \ldots \times \mathbb{P}\left(\mathbb{K}^{A_{n}{ }^{*}}\right)$. We then define an invertible sheaf $\mathcal{L}_{i}$ on $X$ as the inverse image of the sheaf $\mathcal{O}(1)$ from the factor $\mathbb{P}\left(\mathbb{K}^{A_{i}{ }^{*}}\right)$, and set $V_{i}=H^{0}\left(X, \mathcal{L}_{i}\right)$. If we suppose that each $A_{i}$ generates $\mathbb{R}^{n}$ as an affine space and that all $A_{i}$ together generate $\mathbb{Z}^{n}$ as an affine lattice, then the resultant $\operatorname{Res}_{V_{0}, \ldots, V_{n}}$ is well defined (see [GKZ94] VIII.1). Its degree with respect to each $f_{i}$ is the generic number of solutions of the system $\left\{f_{0}=0, \ldots, f_{i-1}=0, f_{i+1}=0, \ldots, f_{n}=0\right\}$. By the BKK theorem [Ber75], this is the mixed volume of $\left\{A_{j}\right\}_{j \neq i}$, that is the coefficient of $\prod_{j \neq i} \lambda_{j}$ in $\operatorname{Vol}\left(\sum_{j \neq i} \lambda_{i} A_{i}\right)=\operatorname{MV}\left(\left\{A_{j}\right\}_{j \neq i}\right) \prod_{j \neq i} \lambda_{j}+\cdots$ where Vol denotes the usual Euclidean volume.

The methods for constructing a Sylvester-type matrix are based on geometric properties of the supports $A_{i}$ (see $[\mathbf{C P 9 3}],[\mathbf{C E 9 3}]$ ). They use the following scheme: for any polytope $A \subset \mathbb{Z}^{n}$ and for any non-zero vector $\delta \in \mathbb{R}^{n}$, let $A^{\delta}$ denotes the set of integer points of $A$ which are not on facets $F$ of $A$ such that the scalar product $n_{F} \cdot \delta>0$, where $n_{F}$ is the exterior normal vector of $F$. Consider now the following (well-defined) linear transformation

$$
\begin{aligned}
\widetilde{\mathcal{S}}:\left\langle\mathbf{t}^{E_{0}}\right\rangle \times \cdots \times\left\langle\mathbf{t}^{E_{n}}\right\rangle & \rightarrow\left\langle\mathbf{t}^{F}\right\rangle \\
\left(q_{0}, \ldots, q_{n}\right) & \mapsto \sum_{i=0}^{n} q_{i} f_{i}
\end{aligned}
$$

where $E_{i}=\left(\oplus_{i \neq j} A_{i}\right)^{\delta}, F=A^{\delta}$. Exploiting the properties of a regular triangulation of $A$, it is possible to extract from $\widetilde{\mathcal{S}}$ a maximal square matrix $\mathrm{S}(\mathbf{c})$, such that, for a sufficiently generic vector $\delta$, its determinant is not generically 0 and such that its degree in the coefficients of $f_{0}$ is exactly the mixed volume of $A_{1}, \ldots, A_{n}$. Therefore, this determinant is a non-trivial multiple of $\operatorname{Res}_{X}\left(\mathbf{f}_{\mathbf{c}}\right)$, the extraneous factor depending only on the coefficients of $f_{1}, \ldots, f_{n}$. A Macaulay-like formula is given in [D'A02], for the explicit description of this extraneous factor for special lifting functions, used to construct a regular subdivision of $A$. We mention also the recent work [Khe02] giving a square matrix for unmixed bivariate systems.

3.4. Residual resultant. In many situations coming from practical problems, the polynomial system has commons zeroes which are independent of the 
parameters, and which we are not interested in. We are going to present here how to compute the resultant in such a situation, under suitable assumptions.

Let $g_{1}, \ldots, g_{r}$ be $r$ homogeneous polynomials of degree $k_{1} \geq \ldots \geq k_{r} \geq 1$ in $S=\mathbb{K}\left[x_{0}, \ldots, x_{n}\right]$, and denote by $G$ the ideal they generate. Being given $n+1$ integers $d_{0} \geq \ldots \geq d_{n}$ greater or equal to $k_{1}$, we would like to compute the resultant associated to the system

$$
\mathbf{f}_{\mathbf{c}}:=\left\{\begin{aligned}
f_{0}(\mathbf{x}) & =\sum_{i=1}^{r} h_{i, 0}(\mathbf{x}) g_{i}(\mathbf{x}) \\
\vdots & \\
f_{n}(\mathbf{x}) & =\sum_{i=1}^{r} h_{i, n}(\mathbf{x}) g_{i}(\mathbf{x})
\end{aligned}\right.
$$

where $h_{i, j}(\mathbf{x})=\sum_{|\alpha|=d_{j}-k_{i}} c_{\alpha}^{i, j} \mathbf{x}^{\alpha}$ is a homogeneous polynomial of degree $d_{j}-k_{i}$. For this we set $X=\mathbb{P}^{n}$, and $V_{i}=H^{0}\left(X, \mathcal{G}\left(d_{i}\right)\right)$ for all $i=0, \ldots, n$, where $\mathcal{G}$ is the coherent ideal sheaf associated to $G$. The vector space $V_{i}$ parameterizes all the homogeneous polynomials of degree $d_{i}$ which are in the saturation of $G$.

Proposition 3.4. ([BEM01]) Suppose that $G$ is a (projective) local complete intersection, and that $d_{n} \geq k_{r}+1$. Then $\operatorname{Res}_{V_{0}, \ldots, V_{n}}$ is well defined and satisfies

$$
\begin{aligned}
\operatorname{Res}_{V_{0}, \ldots, V_{n}}\left(f_{0}, \ldots, f_{n}\right)=0 & \Leftrightarrow F^{\text {sat }} \neq G^{\text {sat }} \\
& \Leftrightarrow\left(F^{\text {sat }}: G^{\text {sat }}\right) \neq S \\
& \Leftrightarrow \mathcal{Z}(F: G) \neq \emptyset
\end{aligned}
$$

where both ideals $F^{\text {sat }}$ and $G^{\text {sat }}$ denote respectively the saturations of the ideals $F=\left(f_{0}, \ldots, f_{n}\right)$ and $G$.

From a geometrical point of view, the vanishing condition can be stated in the blow-up $\tilde{X}$ of $X$ along the ideal sheaf $\mathcal{G}$, that we denote by $\pi: \tilde{X} \rightarrow X$. We have

$$
\operatorname{Res}_{V_{0}, \ldots, V_{n}}\left(f_{0}, \ldots, f_{n}\right)=0 \Leftrightarrow \exists x \in \tilde{X}: \tilde{f}_{i}(x)=0 \forall i \in\{0, \ldots, n\},
$$

where $\tilde{f}_{i}$ denotes the section $\pi^{*}\left(f_{i}\right) \in H^{0}\left(X, \pi^{-1} \mathcal{G} \cdot \mathcal{O}_{\tilde{X}} \otimes \pi^{*}\left(\mathcal{O}_{X}\left(d_{i}\right)\right)\right)$, i.e. the virtual transform of $f_{i}$ by $\pi$. In particular, if there exists a point $x \in X \backslash \mathcal{Z}(G)$ such that $f_{i}(x)=0$ for all $i=0, \ldots, n$, then we deduce that $\operatorname{Res}_{V_{0}, \ldots, V_{n}}\left(f_{0}, \ldots, f_{n}\right)=0$.

The explicit computation of $\operatorname{Res}_{V_{0}, \ldots, V_{n}}$ is known in two cases : the case where $G$ is supposed to be a complete intersection [BEM01] (see also [BKM90, CU00, Bus01a]), and the case where $G$ is supposed to be a (projective) local complete intersection codimension 2 arithmetically Cohen-Macaulay (abbreviated ACM) ideal [Bus01a]. Since we are interested in applications to CAGD, we present only the second case which was originally designed for surface implicitization, taking $X=\mathbb{P}^{2}$ [Bus01b] (point out that a saturated ideal in $\mathbb{P}^{2}$ of codimension 2 is $\mathrm{ACM})$.

The hypothesis $G$ is ACM of codimension 2 is made to have, using HilbertBurch theorem (see [Eis94] theorem 20.15), the following free resolution of the ideal $G$ :

$$
0 \rightarrow \bigoplus_{i=1}^{r-1} S\left[-l_{i}\right] \stackrel{\psi}{\longrightarrow} \bigoplus_{i=1}^{r} S\left[-k_{i}\right] \stackrel{\gamma=\left(g_{1}, \ldots, g_{r}\right)}{\longrightarrow} G \rightarrow 0
$$

with $\sum_{i=1}^{r-1} l_{i}=\sum_{i=1}^{r} k_{i}$. It follows that the Eagon-Northcott complex associated to the graded map

$$
\bigoplus_{i=1}^{r-1} S\left[-l_{i}\right] \bigoplus_{i=0}^{n} S\left[-l_{i}\right] \stackrel{\psi \oplus \phi}{\longrightarrow} \bigoplus_{i=1}^{r} S\left[-k_{i}\right],
$$


where $\phi$ is the matrix $\left(h_{i, j}\right)_{1<i<r, 0 \leq j \leq n}$ resolves the ideal $(F: G)$, and hence the determinants of some of its graded parts are exactly $\operatorname{Res}_{V_{0}, \ldots, V_{n}}$. This result gives a first algorithm to compute $\operatorname{Res}_{V_{0}, \ldots, V_{n}}$, and also its multi-degree as an Euler characteristic. A closed formula for all $n$ is difficult to state, but we can do the computation "by hand" in the useful case of $\mathbb{P}^{2}$, and we obtain that $\operatorname{Res}_{V_{0}, V_{1}, V_{2}}$ is homogeneous in the coefficient of each $f_{i}, i=0,1,2$, of degree

$$
\frac{d_{0} d_{1} d_{2}}{d_{i}}-\frac{\sum_{j=1}^{n-1} l_{j}^{2}-\sum_{j=1}^{n} k_{j}^{2}}{2} .
$$

Another consequence of this formulation in terms of determinant of complex is the usual "gcd maximal minors" property of resultants:

THEOREM 3.5. We denote by $\Delta_{i_{1}, \ldots, i_{r}}$ the determinant of the submatrix of the map $\phi \oplus \psi$ corresponding to columns $i_{1}, \ldots, i_{r}$, and by $\alpha_{i_{1}, \ldots, i_{r}}$ its degree. Then, for any $\nu \geq \sum_{i=0}^{n} d_{i}-n\left(k_{r}+1\right)$, the morphism

$$
\begin{aligned}
\partial_{\nu}: \bigoplus_{0 \leq i_{1}<\ldots<i_{r} \leq n} S_{\nu-\alpha_{i_{1}, \ldots, i_{r}}} e_{i_{1}} \wedge \ldots \wedge e_{i_{r}} & \longrightarrow S_{\nu} \\
e_{i_{1}} \wedge \ldots \wedge e_{i_{r}} & \mapsto \Delta_{i_{1} \ldots i_{r}}
\end{aligned}
$$

is surjective if and only if $\mathcal{Z}(F: G)=\emptyset$ (or $\left.F^{\text {sat }}=G^{\text {sat }}\right)$. In this case, all nonzero maximal minors of size $\operatorname{dim}_{\mathbb{K}}\left(S_{\nu}\right)$ of the matrix $\partial_{\nu}$ is a multiple of $\operatorname{Res}_{V_{0}, \ldots, V_{n}}$, and the gcd of all these maximal minors is exactly the residual resultant.

3.5. General residual resultant. We have seen that we can compute the resultant in presence of base points (if the base points locus is a complete intersection or a local complete intersection ACM of codimension 2) with similar algorithms to the ones known for the classical resultant.

We have seen (corollary 2.4) that if $X=\mathbb{P}^{n}$ and $V_{0}, \ldots, V_{n}$ are very ample almost everywhere, we can define its resultant $\operatorname{Res}_{V_{0}, \ldots, V_{n}}$. Let us see now how to compute a non-zero multiple of it (see [BEM00] for more details).

Definition 3.6. The Bezoutian $\Theta_{f_{0}, \ldots, f_{n}}$ of $f_{0}, \ldots, f_{n} \in S$ is the element of $S \otimes_{\mathbb{K}} S$ defined by

$$
\Theta_{f_{0}, \ldots, f_{n}}(\mathbf{t}, \mathbf{z}):=\left|\begin{array}{cccc}
f_{0}(\mathbf{t}) & \theta_{1}\left(f_{0}\right)(\mathbf{t}, \mathbf{z}) & \cdots & \theta_{n}\left(f_{0}\right)(\mathbf{t}, \mathbf{z}) \\
\vdots & \vdots & \vdots & \vdots \\
f_{n}(\mathbf{t}) & \theta_{1}\left(f_{n}\right)(\mathbf{t}, \mathbf{z}) & \cdots & \theta_{n}\left(f_{n}\right)(\mathbf{t}, \mathbf{z})
\end{array}\right|,
$$

where

$$
\theta_{i}\left(f_{j}\right)(\mathbf{t}, \mathbf{z}):=\frac{f_{j}\left(z_{1}, \ldots, z_{i-1}, t_{i}, \ldots, t_{n}\right)-f_{j}\left(z_{1}, \ldots, z_{i}, t_{i+1}, \ldots, t_{n}\right)}{t_{i}-z_{i}} .
$$

Let $\Theta_{f_{0}, \ldots, f_{n}}(\mathbf{t}, \mathbf{z})=\sum \theta_{\alpha \beta} \mathbf{t}^{\alpha} \mathbf{z}^{\beta}, \theta_{\alpha, \beta} \in \mathbb{K}$. The Bezoutian matrix of $f_{0}, \ldots, f_{n}$ is the matrix $B_{f_{0}, \ldots, f_{n}}=\left(\theta_{\alpha \beta}\right)_{\alpha, \beta}$.

The Bezoutian was used by E. Bézout to construct the resultant of two polynomials in one variable [B.6́4]. In the multivariate case, we have the following property.

THEOREM 3.7. Assume that each $V_{i}$ is very ample almost everywhere, then any maximal minor of the Bezoutian matrix $B_{f_{0}, \ldots, f_{n}}$ is divisible by the resultant $\operatorname{Res}_{V_{0}, \ldots, V_{m}}\left(f_{0}, \ldots, f_{n}\right)$. 
REMARK 3.8. As we said at the end of section 2, it is possible to use other birational transformations than blowing-up to define the resultant. For instance, in [BEM00] it was proved that this general residual resultant can also be constructed with any birational morphism from a dense open subset of $X$ to a projective space. This point of view generalizes monomial parameterizations used to define the toric resultant to polynomial parameterizations. We refer to [BEM00] for more details and conditions similar to "very ampleness almost everywhere".

\section{Surface implicitization}

Algebraic surfaces are basic ingredients in computer aided geometric design. They appear in two forms: parametric and/or implicit representations. The parametric one is given by a generically finite map

$$
\sigma:\left(t_{1}, t_{2}\right) \in U \subset \mathbb{K}^{2} \mapsto\left(\frac{f_{1}\left(t_{1}, t_{2}\right)}{f_{0}\left(t_{1}, t_{2}\right)}, \frac{f_{2}\left(t_{1}, t_{2}\right)}{f_{0}\left(t_{1}, t_{2}\right)}, \frac{f_{3}\left(t_{1}, t_{2}\right)}{f_{0}\left(t_{1}, t_{2}\right)}\right) \in \mathbb{K}^{3}
$$

where the $f_{i}$ are polynomials in $t_{1}$ and $t_{2}$, and $U$ is a subset of $\mathbb{K}^{2}$ such that $f_{0} \neq 0$ on $U$. The implicit equation is given by an irreducible polynomial in three variables $P\left(x_{1}, x_{2}, x_{3}\right)$ of minimal degree such that $P \circ \sigma=0$. Both representations are important for different reasons. The parametric is useful to generate points (and so to draw the surface), the implicit is convenient to test whether a point is in the given surface or not and for intersection purposes.

From a mathematical point of view it is more practice to work whit implicit equations but most surfaces in CAGD are given by parametric representations. So we will focus on the surface implicitization problem, that is, computing the implicit equation of the surface from its parametric representation.

We consider the projective map

$$
\begin{aligned}
\sigma^{h}: \mathbb{P}^{2} \backslash V & \rightarrow \mathbb{P}^{3} \\
\left(t_{0}: t_{1}: t_{2}\right) & \mapsto\left(f_{0}^{h}(\mathbf{t}): f_{1}^{h}(\mathbf{t}): f_{2}^{h}(\mathbf{t}): f_{3}^{h}(\mathbf{t})\right),
\end{aligned}
$$

where $\mathbf{t}=\left(t_{0}: t_{1}: t_{2}\right), f_{0}^{h}, \ldots, f_{3}^{h}$ are the homogenization of $f_{0}, \ldots, f_{3}$ (with respect to $\left.t_{0}\right)$ of degree $d=\max _{i=0, \ldots, 3}\left(\operatorname{deg}\left(f_{i}\right)\right)$, and $V$ is the zero-locus of $f_{0}^{h}=$ $\cdots=f_{3}^{h}=0$ in $\mathbb{P}^{2}$. The graph of $\sigma$ can be described by the equations

$$
\left\{\begin{array}{l}
f_{1}(\mathbf{t})-x_{1} f_{0}(\mathbf{t})=0 \\
f_{2}(\mathbf{t})-x_{2} f_{0}(\mathbf{t})=0 \\
f_{3}(\mathbf{t})-x_{3} f_{0}(\mathbf{t})=0
\end{array}\right.
$$

where $x_{1}, x_{2}, x_{3}$ denotes affine coordinates in $\mathbb{P}^{3}$. Computing the implicit equation reduces to eliminate the variables $\mathbf{t}$ in this system. The result is the equation of the closed image of $\sigma^{h}$, which is of the form $P\left(x_{1}, x_{2}, x_{3}\right)^{\beta}$, where $\beta$ is the degree of the map $\sigma$ (the number of points in a generic fiber of $\sigma^{h}$ onto its image).

In the following subsections we will list some methods to compute the implicit equation, using resultants with/without base points (a base point being a point in $V)$ or the syzygies of the polynomials $f_{0}^{h}, f_{1}^{h}, f_{2}^{h}, f_{3}^{h}$.

4.1. The implicit equation as an anisotropic resultant. As far as we know, the best way to compute the implicit equation $P$ of $\sigma$ if $\sigma^{h}$ has no base points is to use the anisotropic resultant. We denote by $x_{0}$ the homogenization variable in $\mathbb{P}^{3}$. The basic idea of the following proposition is that a point $\left(x_{0}: x_{1}\right.$ : 
$\left.x_{2}: x_{3}\right) \in \mathbb{P}^{3}$ is on $P$ if and only if there exists a point $\mathbf{t}=\left(t_{0}: t_{1}: t_{2}\right) \in \mathbb{P}^{2}$ and a non-zero scalar $\lambda$ such that

$$
\left\{\begin{array}{l}
\lambda x_{0}-f_{0}^{h}(\mathbf{t})=0, \\
\lambda x_{1}-f_{1}^{h}(\mathbf{t})=0, \\
\lambda x_{2}-f_{2}^{h}(\mathbf{t})=0, \\
\lambda x_{3}-f_{3}^{h}(\mathbf{t})=0 .
\end{array}\right.
$$

Introducing a new variable $\lambda$ of weight $d$, so that equations $\lambda x_{i}-f_{i}^{h}(\mathbf{t})$ are isobar of degree $d$, we have:

THEOREM 4.1. ([Jou96] 5.3.17) Assume that $\sigma^{h}$ has no base points, and that $\operatorname{deg}(\lambda)=d, \operatorname{deg}\left(t_{0}\right)=\operatorname{deg}\left(t_{1}\right)=\operatorname{deg}\left(t_{2}\right)=1$. Then the anisotropic resultant

${\operatorname{Res} a \mathbb{P}^{4}}\left(\lambda x_{0}-f_{0}^{h}(\mathbf{t}), \lambda x_{1}-f_{1}^{h}(\mathbf{t}), \lambda x_{2}-f_{2}^{h}(\mathbf{t}), \lambda x_{3}-f_{3}^{h}(\mathbf{t})\right)=P\left(x_{0}, x_{1}, x_{2}, x_{3}\right)^{\beta}$, where $\beta$ is the degree of $\sigma^{h}$. It is of total degree $d^{2}$.

Jouanolou gives some square matrices to compute explicitly this anisotropic resultant in [Jou96], which have the advantage to yield a generic formula for all the parameterized surfaces without base points. Notice that these matrices were rediscovered, in some particular cases, in [AS01].

The usual construction used to solve the surface implicitization problem without base points and based on classical resultants is given by the following theorem. We give a proof (that we did not find in the literature) of it, based on the properties of resultants.

TheOrem 4.2. Assume that $\sigma^{h}$ has no base points. If $\beta$ is the degree of $\sigma^{h}$, then the classical resultant

$$
\operatorname{Res}_{\mathbb{P} 3}\left(x_{1} f_{0}^{h}(\mathbf{t})-f_{1}^{h}(\mathbf{t}), x_{2} f_{0}^{h}(\mathbf{t})-f_{2}^{h}(\mathbf{t}), x_{3} f_{0}^{h}(\mathbf{t})-f_{3}^{h}(\mathbf{t})\right)=P\left(1, x_{1}, x_{2}, x_{3}\right)^{\beta} .
$$

It is of total degree (in $\mathbf{x}) d^{2}$.

Proof. We introduce a new variable $\lambda$ and consider the classical resultant :

$$
\mathcal{R}(\mathbf{x}):=\operatorname{Res}_{\mathbb{P}^{4}}\left(\lambda^{d} x_{0}-f_{0}^{h}(\mathbf{t}), \lambda^{d} x_{1}-f_{1}^{h}(\mathbf{t}), \lambda^{d} x_{2}-f_{2}^{h}(\mathbf{t}), \lambda^{d} x_{3}-f_{3}^{h}(\mathbf{t})\right) .
$$

By lemma 3.1, $\mathcal{R}$ is isobar of degree $d^{4}$, assuming that $\operatorname{deg}\left(x_{i}\right)=d$ for all $i=$ $0,1,2,3$. It follows that $\mathcal{R}$ is a homogeneous polynomial in $\mathbb{K}\left[x_{0}, x_{1}, x_{2}, x_{3}\right]$ of degree $d^{3}$. Moreover it is clear that $\mathcal{R}$ vanishes at a point $\left(x_{0}: x_{1}: x_{2}: x_{3}\right)$ if and only if this point is in the surface $S$, and we deduce, since $\operatorname{deg}(S)=d^{2}$, that $\mathcal{R}=S^{d}$ (this also follows from lemma 3.3). In order to recover the classical resultant over $\mathbb{P}^{3}$, we "localize" the preceding formula using the Poisson's formula (see lemma 3.2) as follows.

After the specialization of $x_{0}$ to 1 , by classical invariance properties we have of the resultant

$$
\begin{aligned}
\mathcal{R} & =\operatorname{Res}_{\mathbb{P}^{4}}\left(\lambda^{d}-f_{0}^{h}, \lambda^{d} x_{1}-f_{1}^{h}-x_{1}\left(\lambda^{d}-f_{0}^{h}\right), \ldots, \lambda^{d} x_{3}-f_{3}^{h}-x_{3}\left(\lambda^{d}-f_{0}^{h}\right)\right) \\
& =\operatorname{Res}_{\mathbb{P}^{4}}\left(\lambda^{d}-f_{0}^{h}, x_{1} f_{0}^{h}-f_{1}^{h}, x_{2} f_{0}^{h}-f_{2}^{h}, x_{3} f_{0}^{h}-f_{3}^{h}\right),
\end{aligned}
$$

and the Poisson's formula gives

$$
\mathcal{R}=\operatorname{det}\left(M_{1-f_{0}^{h}(\mathbf{t})}\right) \operatorname{Res}_{\mathbb{P} 3}\left(x_{1} f_{0}^{h}-f_{1}^{h}, x_{2} f_{0}^{h}-f_{2}^{h}, x_{3} f_{0}^{h}(\mathbf{t})-f_{3}^{h}\right)^{d},
$$

where $\operatorname{det}\left(M_{1-f_{0}^{h}(\mathbf{t})}\right)$ denotes the determinant of the multiplication by $1-f_{0}^{h}(\mathbf{t})$ modulo the polynomials $x_{1} f_{0}^{h}(\mathbf{t})-f_{1}^{h}(\mathbf{t}), x_{2} f_{0}^{h}-f_{2}^{h}, x_{3} f_{0}^{h}(\mathbf{t})-f_{3}^{h}(\mathbf{t})$. To conclude the proof, we can either compute this last determinant (and find 1), or remark that the resultant $\operatorname{Res}_{\mathbb{P} 3}\left(x_{1} f_{0}^{h}-f_{1}^{h}, x_{2} f_{0}^{h}-f_{2}^{h}, x_{3} f_{0}^{h}(\mathbf{t})-f_{3}^{h}\right)$ is a power of $P$ (this resultant vanishes at $\mathbf{x}$ if and only if $\mathbf{x}$ is on $S$ ) and compare the degrees. 
4.2. The implicit equation as a residual resultant. In section 4.1 we saw that classical and anisotropic resultants yield the implicit equation we are looking for, provided $\sigma^{h}$ has no base points. If there exists base points, then both resultants vanish identically since system (5) have solutions independent of parameters $x_{0}, \ldots, x_{3}$. In this context, we have to consider residual resultants to "erase" the base points (section 3.4). We suppose hereafter that the base points are in codimension 2 , this is not restrictive for base points of pure codimension 1 can be erased by a single gcd computation.

Let $G=\left(g_{1}, \ldots, g_{r}\right)$ be a saturated projective local complete intersection of codimension 2 in $\mathbb{P}^{2}$; this is the ideal of base points. We denote by $k_{1} \geq \ldots \geq k_{r} \geq$ 1 the respective degree of the polynomials $g_{1}, \ldots, g_{r}$. Since $G$ is saturated, it is $\mathrm{ACM}$, and hence satisfies the hypothesis of 3.4. Recall that $G$ have the following free resolution

$$
0 \rightarrow \bigoplus_{i=1}^{r-1} \mathbb{K}\left[t_{0}, t_{1}, t_{2}\right]\left(-l_{i}\right) \stackrel{\psi}{\longrightarrow} \bigoplus_{i=1}^{r} \mathbb{K}\left[t_{0}, t_{1}, t_{2}\right]\left(-k_{i}\right) \stackrel{\gamma=\left(g_{1}, \ldots, g_{r}\right)}{\longrightarrow} G \rightarrow 0,
$$

from which we deduce that $\sum_{p \in V(G)} d_{p}$, where $d_{p}$ denotes the multiplicity of the point $p \in V(G)$, is exactly

$$
\sum_{p \in V(G)} d_{p}=\frac{\sum_{j=1}^{n-1} l_{j}^{2}-\sum_{j=1}^{n} k_{j}^{2}}{2}
$$

Let $V=H^{0}\left(\mathbb{P}^{2}, \mathcal{G}(d)\right)$, where $\mathcal{G}$ is the ideal sheaf associated to $G$, the vector space parameterizing all homogeneous polynomials of degree $d$ in the ideal $G$.

Proposition 4.3. ([Bus01b] theorem 3.2) Suppose that $d \geq k_{1}, d \geq k_{r}+1$ and let $F=\left(f_{0}, f_{1}, f_{2}, f_{3}\right)$. If $F^{\text {sat }}=G$, that is $G$ define the base points of the parameterization given by $F$, then the residual resultant

$$
\operatorname{Res}_{\oplus_{i=1}^{3} V}\left(f_{1}-x_{1} f_{0}, f_{2}-x_{2} f_{0}, f_{3}-x_{3} f_{0}\right)=P\left(1, x_{1}, x_{2}, x_{3}\right)^{\beta},
$$

where $P$ is the implicit equation of $\sigma$ and $\beta$ its degree. It is of total degree $d^{2}-$ $\sum_{p \in V(G)} d_{p}$.

EXAMPLE 4.4. Let

$$
\left\{\begin{array}{l}
f_{0}^{h}=t_{0}^{2} t_{1}+t_{0}^{2} t_{2}+t_{0} t_{1} t_{2}+t_{1} t_{2}^{2} \\
f_{1}^{h}=t_{0} t_{1}^{2}+t_{0}^{2} t_{2}+t_{0} t_{1} t_{2}+t_{0} t_{2}^{2} \\
f_{2}^{h}=t_{0}^{2} t_{1}+t_{0} t_{1}^{2}+t_{0} t_{1} t_{2}+t_{0} t_{2}^{2}+t_{1} t_{2}^{2} \\
f_{3}^{h}=t_{0}^{2} t_{2}+t_{0} t_{1} t_{2}+t_{1}^{2} t_{2}+t_{0} t_{2}^{2}+t_{1} t_{2}^{2}
\end{array}\right.
$$

Erasing base points defined by the ideal $G=\left(t_{0} t_{1}, t_{0} t_{2}, t_{1} t_{2}\right)$, we find $a 10 \times 10$ matrix whose determinant is the implicit equation of degree 6 :

$-17 x_{1}^{6}-76 x_{1}^{5} x_{2}-169 x_{1}^{4} x_{2}^{2}-248 x_{1}^{3} x_{2}^{3}-261 x_{1}^{2} x_{2}^{4}-176 x_{1} x_{2}^{5}-\cdots-27 x_{1}+27 x_{2}-27 x_{3}$.

We point out that this method for computing implicit equation has the disadvantage to require the knowledge of the base points, but has the advantage to yield universal formulas (as always with resultant-based methods) for all surfaces having the same base points.

Note that if the ideal $F=\left(f_{0}, f_{1}, f_{2}, f_{3}\right)$ is saturated, then the preceding method do not work. In fact, in this case, a classical resultant of the syzygies of $F$ gives the implicit equation, as we will see later. 
4.3. Moving quadrics. The method of moving quadrics was introduced in [SC95] in order to compute the implicit equation of a given parameterized surface. Its main ingredients are moving planes and moving quadrics which follow the surface. A moving plane of degree $\nu \in \mathbb{N}$ following the surface is a polynomial in $\mathbb{K}\left[t_{0}, t_{1}, t_{2}\right]\left[x_{0}, x_{1}, x_{2}\right]$ of the form

$$
a_{0}\left(t_{0}, t_{1}, t_{2}\right) x_{0}+a_{1}\left(t_{0}, t_{1}, t_{2}\right) x_{1}+a_{2}\left(t_{0}, t_{1}, t_{2}\right) x_{2}+a_{3}\left(t_{0}, t_{1}, t_{2}\right) x_{3},
$$

where $a_{0}, a_{1}, a_{2}$ and $a_{3}$ are homogeneous polynomials in $\mathbb{K}\left[t_{0}, t_{1}, t_{2}\right]$ of degree $\nu$, such that it vanishes if we replace each $x_{i}$ by $f_{i}^{h}$. This is equivalent to say that $\left(a_{0}, a_{1}, a_{2}, a_{3}\right)$ is a first syzygy of the ideal $\left(f_{0}^{h}, f_{1}^{h}, f_{2}^{h}, f_{3}^{h}\right)$ of degree $\nu$. Similarly a moving quadric of degree $\nu \in \mathbb{N}$ following the surface is a polynomial in $\mathbb{K}\left[t_{0}, t_{1}, t_{2}\right]\left[x_{0}, x_{1}, x_{2}\right]$ of the form

$$
\sum_{0 \leq i \leq j \leq 3} a_{i, j}\left(t_{0}, t_{1}, t_{2}\right) x_{i} x_{j}=a_{0,0}\left(t_{0}, t_{1}, t_{2}\right) x_{0}^{2}+\ldots+a_{3,3}\left(t_{0}, t_{1}, t_{2}\right) x_{3}^{2},
$$

where the $a_{i, j}$ 's are homogeneous polynomials in $\mathbb{K}\left[t_{0}, t_{1}, t_{2}\right]$ of degree $\nu$, such that it vanishes if we replace each $x_{i}$ by $f_{i}^{h}$. This is also equivalent to say that $\left(a_{0,0}, a_{0,1}, \ldots, a_{3,3}\right)$ is a first syzygy of the ideal $\left(f_{0}^{h}, f_{1}^{h}, f_{2}^{h}, f_{3}^{h}\right)^{2}$ of degree $\nu$. Let us choose $p$ moving planes $L_{1}, \ldots, L_{p}$ of degree $\nu$ that we rewrite as

$$
L_{j}=\sum_{i=0}^{3} a_{i}\left(t_{0}, t_{1}, t_{2}\right) x_{i}=\sum_{|\alpha|=\nu} l_{j, \alpha}\left(x_{0}, x_{1}, x_{2}, x_{3}\right) \mathbf{t}^{\alpha},
$$

where each $l_{j, \alpha}\left(x_{0}, x_{1}, x_{2}, x_{3}\right)$ is a linear form in $\mathbb{K}\left[x_{0}, x_{1}, x_{2}, x_{3}\right]$. Let us consider also $q$ moving quadrics $Q_{1}, \ldots, Q_{q}$ of the same degree $\nu$ that we rewrite as

$$
Q_{s}=\sum_{0 \leq i \leq j \leq 3} a_{i, j}\left(t_{0}, t_{1}, t_{2}\right) x_{i} x_{j}=\sum_{|\alpha|=\nu} q_{s, \alpha}\left(x_{0}, x_{1}, x_{2}, x_{3}\right) \mathbf{t}^{\alpha},
$$

where each $q_{j, \alpha}\left(x_{0}, x_{1}, x_{2}, x_{3}\right)$ is a homogeneous polynomial of degree 2 in the polynomial ring $\mathbb{K}\left[x_{0}, x_{1}, x_{2}, x_{3}\right]$. Assuming that $p+q=\left(\begin{array}{c}\nu+2 \\ 2\end{array}\right)$ we can define the following square matrix

$$
\left(\begin{array}{cccccc}
l_{1, \alpha_{1}} & \cdots & l_{p, \alpha_{1}} & q_{1, \alpha_{1}} & \cdots & q_{q, \alpha_{1}} \\
\vdots & & \vdots & \vdots & & \vdots \\
l_{1, \alpha_{p+q}} & \cdots & l_{p, \alpha_{p+q}} & q_{1, \alpha_{p+q}} & \cdots & q_{q, \alpha_{p+q}}
\end{array}\right),
$$

where $\alpha_{1}, \ldots, \alpha_{p+q}$ correspond to the $p+q$ monomials of degree $\nu$ in variables $t_{0}, t_{1}, t_{2}$. The determinant of this matrix is then a homogeneous polynomial of degree $p+2 q$ (possibly 0 ) in variables $x_{0}, x_{1}, x_{2}, x_{3}$. This polynomial is easily seen to vanish on the parameterised surface $P$ by construction. The key point of the moving surface method is to prove that we can find $p$ moving planes and $q$ moving quadrics such that the determinant of (6) is non-zero and such that $p+2 q$ equals the degree of $P$. In case the parameterization where $f_{0}^{h}, f_{1}^{h}, f_{2}^{h}, f_{3}^{h}$ has no base points and $\sigma$ is birational, this was proved in [CGZ00] with $\nu=d-1$. D'Andréa proved the general case without base points :

Theorem 4.5. ([D'A01] corollary 5.3) Suppose that $\sigma^{h}$ has no base points and that there are $d$ moving planes of degree $d-1$ following the surface. Then we can construct a matrix of type (6) with $\nu=d-1$ whose determinant is the implicit equation of $\sigma$ to its degree. 
The case where base points are present was recently investigated in [BCD02], assuming that $\sigma^{h}$ is birational. The main needed hypothesis is that the ideal of the parameterization is a local complete intersection, but some other technical hypothesis are necessary.

TheOREM 4.6. ([BCD02] theorem 3.6) Suppose that $\sigma^{h}$ is birational and that

i) $f_{0}^{h}, f_{1}^{h}, f_{2}^{h}, f_{3}^{h}$ are linearly independent over $\mathbb{K}$,

ii) $V\left(f_{0}^{h}, f_{1}^{h}, f_{2}^{h}, f_{3}^{h}\right)$ consists of a finite number of points and is a local complete intersection,

iii) There is $n \in\{d-1, d-2\}$ such that $\operatorname{dim}_{\mathbb{C}}(R / I)_{n+d}=\operatorname{deg}(V(I))$ which is the number of base points counted with multiplicity,

iv) $f_{3}^{h} \in\left(f_{0}^{1}, f_{1}^{h}, f_{2}^{h}\right)^{s a t}$,

v) There is no syzygy of degree $n$ of $f_{0}^{1}, f_{1}^{h}, f_{2}^{h}$, where $n$ is as in iii).

Then we can construct a matrix of type (6) with $\nu=n$ whose determinant is the closed image of $\sigma^{h}$.

In [BCD02] it was also proved that this method fails in particular cases. For instance if a parameterization satisfies all the hypothesis of the previous theorem except v), then the method fails (see [BCD02], remark 3.11). When the ideal $F=\left(f_{0}^{h}, f_{1}^{h}, f_{2}^{h}, f_{3}^{h}\right)$ of the parameterization is saturated, this method is also quite limited: if $I$ is saturated and satisfies the hypothesis of the previous theorem, then the method works only for $\nu=d-2$ and $d \leq 3$ (see [BCD02], proposition 4.1). However, in this particular case we can compute the implicit equation as the classical resultant of the syzygies of $F$. Assuming $F$ saturated, there exists an exact complex

$$
0 \rightarrow \bigoplus_{i=1}^{3} \mathbb{K}\left[t_{0}, t_{1}, t_{2}\right]\left(-l_{i}\right) \stackrel{A}{\longrightarrow} \bigoplus_{i=1}^{4} \mathbb{K}\left[t_{0}, t_{1}, t_{2}\right](-d) \stackrel{\gamma=\left(f_{0}^{h}, \ldots, f_{3}^{h}\right)}{\longrightarrow} F \rightarrow 0,
$$

where $A$ is given by a matrix

$$
A=\left(\begin{array}{lll}
p_{1} & q_{1} & r_{1} \\
p_{2} & q_{2} & r_{2} \\
p_{3} & q_{3} & r_{3} \\
p_{4} & q_{4} & r_{4}
\end{array}\right)
$$

Proposition 4.7. ([BCD02] theorem 4.3) Suppose that $F$ is a saturated local complete intersection ideal, then the classical resultant

$\operatorname{Res}_{\mathbb{P}^{2}}\left(p_{1} x_{0}+p_{2} x_{1}+p_{3} x_{2}+p_{4} x_{3}, q_{1} x_{0}+q_{2} x_{1}+q_{3} x_{2}+q_{4} x_{3}, r_{1} x_{0}+r_{2} x_{1}+r_{3} x_{2}+r_{4} x_{3}\right)$ defines the implicit equation of $\sigma$ to its generic degree.

4.4. Approximation complexes. Another method based on syzygies computations has been introduced to solve the surface implicitization problem in [BJ02]. In fact this method is quite closed to the method of moving surfaces but uses only moving planes. The implicit equation is here generally obtained as the determinant of certain complexes that we now describe.

First we denote by $K_{\bullet}(\mathbf{x})$ the exact Koszul complex associated to the regular sequence $\left(x_{0}, x_{1}, x_{2}, x_{3}\right)$ in $\mathbb{K}[\mathbf{x}]:=\mathbb{K}\left[x_{0}, x_{1}, x_{2}, x_{3}\right]$ :

$$
\left(K_{\bullet}(\mathbf{x}), d_{\bullet}^{\mathbf{x}}\right): 0 \rightarrow \mathbb{K}[\mathbf{x}] \stackrel{d_{4}^{\mathbf{x}}}{\longrightarrow} \mathbb{K}[\mathbf{x}]^{4} \stackrel{d_{3}^{\mathbf{x}}}{\longrightarrow} \mathbb{K}[\mathbf{x}]^{6} \stackrel{d_{2}^{\mathbf{x}}}{\longrightarrow} \mathbb{K}[\mathbf{x}]^{4} \stackrel{d_{1}^{\mathbf{x}}=\left(x_{0}, x_{1}, x_{2}, x_{3}\right)}{\longrightarrow} \mathbb{K}[\mathbf{x}] .
$$

Consider now the Koszul complex, that we will denote by $\left(K_{\bullet}(\mathbf{f}), d_{\bullet}^{\mathbf{f}}\right)$, associated to the sequence $\left(f_{0}^{h}, f_{1}^{h}, f_{2}^{h}, f_{3}^{h}\right)$ in $\mathbb{K}[\mathbf{t}]:=\mathbb{K}\left[t_{0}, t_{1}, t_{2}\right]$, but that we see in the 
extension $\mathbb{K}[\mathbf{t}][\mathbf{x}]:=\mathbb{K}[\mathbf{t}] \otimes_{\mathbb{K}} \mathbb{K}[\mathbf{x}]$ :

$$
0 \rightarrow \mathbb{K}[\mathbf{t}][\mathbf{x}] \stackrel{d_{4}^{\mathrm{f}}}{\longrightarrow} \mathbb{K}[\mathbf{t}][\mathbf{x}]^{4} \stackrel{d_{3}^{\mathrm{f}}}{\longrightarrow} \mathbb{K}[\mathbf{t}][\mathbf{x}]^{6} \stackrel{d_{2}^{\mathrm{f}}}{\longrightarrow} \mathbb{K}[\mathbf{t}][\mathbf{x}]^{4} \stackrel{d_{1}^{\mathrm{f}}=\left(f_{0}^{h}, f_{1}^{h}, f_{2}^{h}, f_{3}^{h}\right)}{\longrightarrow} \mathbb{K}[\mathbf{t}][\mathbf{x}] .
$$

Let $\mathcal{Z}_{i}$ denote the kernel of the differential $d_{i}^{\mathbf{f}}$ of $K_{\bullet}(\mathbf{f})$ for $i=1,2,3,4$. The first approximation complex, also called the $\mathcal{Z}$-approximation complex, of $\left(f_{0}^{h}, f_{1}^{h}, f_{2}^{h}, f_{3}^{h}\right)$ is then defined to be the complex $\left(\mathcal{Z}_{\bullet}, d_{\bullet}^{\mathbf{x}}\right)$. The following diagram is helpful to summarize the situation :

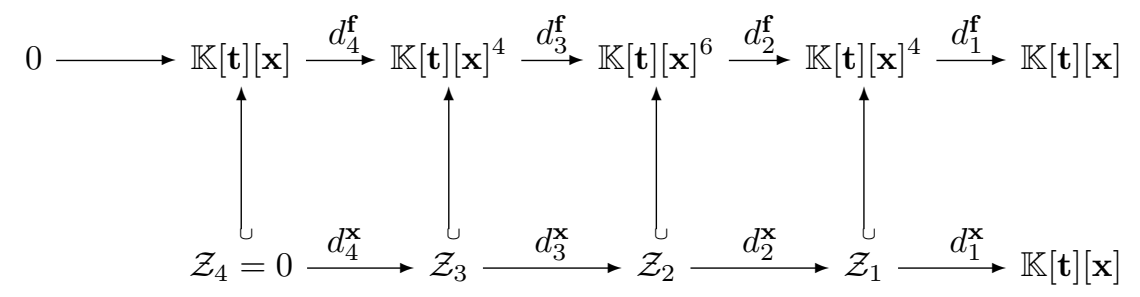

Observe that $\mathcal{Z}_{4}=0$ since $d_{4}^{\mathrm{f}}$ is injective. Each module $\mathcal{Z}_{i}$ can be considered as graded $\mathbb{K}[\mathbf{t}]$-module. We denote by $\mathcal{Z}_{i \nu}$ its graded part of degree $\nu$ : it is a $\mathbb{K}[\mathbf{x}]$ module and $\mathcal{Z}_{i_{\nu}} \hookrightarrow \mathbb{K}[\mathbf{t}]_{\nu} \otimes_{\mathbb{K}} \mathbb{K}[\mathbf{x}]$. An element of $\mathcal{Z}_{1 \nu}$ is an element $\left(a_{0}, a_{1}, a_{2}, a_{3}\right) \in$ $\mathbb{K}[\mathbf{t}][\mathbf{x}]^{4}$ such that $a_{0} f_{0}^{h}+a_{1} f_{1}^{h}+a_{3} f_{3}^{h}+a_{4} f_{4}^{h}=0$, that is a syzygy of $\left(f_{0}^{h}, f_{1}^{h}, f_{2}^{h}, f_{3}^{h}\right)$ in $\mathbb{K}[\mathbf{t}][\mathbf{x}]$. Moreover, we have $d_{1}^{\mathbf{x}}\left(a_{0}, a_{1}, a_{2}, a_{3}\right)=a_{0} x_{0}+a_{1} x_{1}+a_{2} x_{2}+a_{3} x_{3}$, that we called a moving plane of degree $\nu$ as in the previous subsection. We have the following result:

THEOREM 4.8. ([BJ02]) Suppose that $\left(f_{0}^{h}, f_{1}^{h}, f_{2}^{h}, f_{3}^{h}\right)$ defines local complete intersection isolated points (possibly empty). Then for all $\nu \geq 2(d-1)$, the determinant of the $\mathcal{Z}$-approximation complex in degree $\nu$, which is the complex of $\mathbb{K}[\mathbf{x}]$-modules

$$
0 \rightarrow \mathcal{Z}_{3 \nu} \stackrel{d_{3}^{\mathrm{x}}}{\longrightarrow} \mathcal{Z}_{2 \nu} \stackrel{d_{2}^{\mathrm{x}}}{\longrightarrow} \mathcal{Z}_{1 \nu} \stackrel{d_{1}^{\mathrm{x}}}{\longrightarrow} \mathbb{K}[\mathbf{t}]_{\nu} \otimes_{\mathbb{K}} \mathbb{K}[\mathbf{x}] \simeq \mathbb{K}[\mathbf{x}]^{\left({ }^{\nu+2}\right)},
$$

is the implicit equation of $\sigma$ to its degree.

This theorem yields a new algorithm to compute the implicit equation of a parameterized surface having local complete intersection base points, without any other hypothesis. From the known method to compute the determinant of a complex (see the appendix) we deduce that the equation of the surface is obtained as $\frac{\Delta_{0} \Delta_{2}}{\Delta_{1}}$, where $\Delta_{i}$ denotes the determinant of a certain square submatrix of the differential $d_{i}^{\mathrm{x}}$ of $\mathcal{Z}_{\bullet}$. Moreover we have the following corollary :

COROLlaRY 4.9. Under the hypothesis of theorem 4.8, the implicit equation of $\sigma$ to its degree is the gcd of the maximal minors (of size $\left(\begin{array}{c}\nu+2 \\ 2\end{array}\right)$ ) of the surjective matrix $M_{\mathbf{f}, \nu}$ of the map

$$
\begin{aligned}
\mathcal{Z}_{1 \nu} & \stackrel{d_{1}^{\mathrm{x}}}{\longrightarrow} \mathbb{K}[\mathbf{t}]_{\nu} \otimes_{\mathbb{K}} \mathbb{K}[\mathbf{x}] \\
\left(a_{0}, a_{1}, a_{2}, a_{3}\right) & \mapsto a_{0} x_{0}+a_{1} x_{1}+a_{2} x_{2}+a_{3} x_{3} .
\end{aligned}
$$

Consequently, a given point $\left(x_{0}: x_{1}: x_{2}: x_{3}\right) \in \mathbb{P}^{3}$ is on the surface if and only if the rank of $M_{\mathbf{f}, \nu}$ drops.

EXAMPLE 4.10. Let $f_{0}^{h}=s^{3}, f_{1}^{h}=t^{2} u, f_{2}^{h}=s^{2} t+u^{3}$ and $f_{3}^{h}=s t u$. Applying the method we find $\nu=3$ and $M_{\mathbf{f}, 3}$ is a $10 \times 14$ matrix. As a determinant of complex, the implicit equation $-x_{0}^{3} x_{1}^{4}+x_{0}^{2} x_{1}^{3} x_{2} x_{3}-x_{3}^{7}$ is obtained as the product of two determinants of size $10 \times 10$ and $1 \times 1$ divided by another one of size $4 \times 4$. 
4.5. The implicit equation and Bezoutian. In the presence of base points, which do not satisfies some restrictive geometric hypotheses, the methods using classical resultants, the residual resultants and the approximation complexes methods fail. In these cases the Bezoutian (see definition 3.6) can be used to solve the implicitization problem for parametric rational $3 D$-surfaces.

Let $\sigma$ be the parametrization given by (4). From section 3.5, we have

THEOREM 4.11. Every non-zero maximal minor of the Bezoutian matrix of the polynomials $x_{1} f_{0}\left(t_{1}, t_{2}\right)-f_{1}\left(t_{1}, t_{2}\right), x_{2} f_{0}\left(t_{1}, t_{2}\right)-f_{2}\left(t_{1}, t_{2}\right), x_{3} f_{0}\left(t_{1}, t_{2}\right)-f_{3}\left(t_{1}, t_{2}\right)$ in $\mathbb{K}\left[x_{1}, x_{2}, x_{3}\right]\left[t_{1}, t_{2}\right]$ is a multiple of the implicit equation of the image of $\sigma$.

This result is based on the fact that this Bezoutian matrix is a multiple of the multiplication by the first poynomial in the vector space quotiented by the others.

EXAMPLE 4.12 . Let $P$ be the following surface

$$
\left\{\begin{array}{c}
x_{1}=\frac{t_{2}^{2} t_{1}}{\left(1+t_{1}^{2}+t_{2}^{2}\right)}=\frac{t_{2}^{2} t_{1}\left(1+t_{1}^{2}+t_{2}^{2}\right)^{2}}{\left(1+t_{1}^{2}+t_{2}^{2}\right)^{3}} \\
x_{2}=\frac{t_{1}^{2} t_{2}}{\left(1+t_{1}^{2}+t_{2}^{2}\right)^{2}}=\frac{t_{1}^{2} t_{2}\left(1+t_{1}^{2}+t_{2}^{2}\right)}{\left(1+t_{1}^{2}+t_{2}^{2}\right)^{3}} \\
x_{3}=\frac{t_{1} t_{2}}{\left(1+t_{1}^{2}+t_{2}^{2}\right)^{3}}
\end{array}\right.
$$

A maximal minor of the Bezoutian of the polynomials $x_{1} f_{0}-f_{1}, x_{2} f_{0}-f_{2}, x_{3} f_{0}-f_{3}$ in $\mathbb{K}\left[x_{1}, x_{2}, x_{3}\right]\left[t_{1}, t_{2}\right]$ is

$2 x_{2} x_{3}^{3}\left(x_{3}^{6} x_{1}^{10}-6 x_{3}^{5} x_{2}^{3} x_{1}^{7}+6 x_{3}^{4} x_{2}^{4} x_{1}^{6}+2 x_{3}^{6} x_{2}^{2} x_{1}^{6}+2 x_{3}^{3} x_{2}^{7} x_{1}^{5}-9 x_{1}^{5} x_{2}^{3} x_{3}^{5}+9 x_{3}^{4} x_{2}^{6} x_{1}^{4}+6 x_{3}^{4} x_{2}^{4} x_{1}^{4}\right.$ $\left.-6 x_{3}^{5} x_{2}^{5} x_{1}^{3}-x_{3}^{3} x_{2}^{5} x_{1}^{3}-6 x_{3}^{2} x_{2}^{10} x_{1}^{2}+3 x_{3}^{2} x_{2}^{8} x_{1}^{2}+6 x_{3}^{4} x_{2}^{6} x_{1}^{2}+x_{3}^{6} x_{2}^{4} x_{1}^{2}-3 x_{3} x_{2}^{11} x_{1}+2 x_{3}^{3} x_{2}^{9} x_{1}+x_{2}^{14}\right)$.

Separating the extraneous term $x_{2} x_{3}^{3}$ from the implicit equation (without multivariable factorization) can be achieved by using multidimensional Newton formulas ([GV97], [EM02]).

4.6. Toric patches and resultants. Base points appear naturally in rational parameterization such as weighted Bézier parameterization. These parameterization are constructed as follows. Consider control points $p_{i, j} \in \mathbb{K}^{3}$ and weights $w_{i, j}$ for $0 \leq i \leq m_{1}, 0 \leq j \leq m_{2}$. We denote by $\bar{p}_{i, j}=\left(w_{i, j}, w_{i, j} p_{i, j}\right) \in \mathbb{K}^{4} \mathrm{a}$ representative of the corresponding projective point $\in \mathbb{P}^{3}$. Let us defined now the Bézier parameterization

$$
\begin{aligned}
\sigma^{h}: \mathbb{K}^{2}-V & \rightarrow \mathbb{P}^{3} \\
\left(t_{1}, t_{2}\right) & \mapsto \sum_{0 \leq i \leq m_{1}} \sum_{0 \leq j \leq m_{2}} \bar{p}_{i, j} B_{m_{1}}^{i}\left(t_{1}\right) B_{m_{2}}^{j}\left(t_{2}\right)
\end{aligned}
$$

where $B_{n}^{i}(t)=\left(\begin{array}{c}n \\ i\end{array}\right) t^{i}(1-t)^{i}$ and where $V$ is the set of base points, that is the set of parameters $\left(t_{1}, t_{2}\right)$ such that $\sum_{0<i<m_{1}} \sum_{0<j<m_{2}} \bar{p}_{i, j} B_{n}^{i}\left(t_{1}\right) B_{m}^{j}\left(t_{2}\right)=0$.

In the case, where $\bar{p}_{0,0}=0, \bar{p}_{m_{1}, 0}=0, \bar{p}_{0, m_{2}}=0$ or $\bar{p}_{m_{1}, m_{2}}$, the parameterization $\sigma^{h}$ has base points; namely $(0,0),(1,0),(0,1)$ or $(1,1)$. These base points are blown up into curves, which produce multisided Bézier patches, as described in $[?]$.

Such as situation is naturally connected to toric varieties. Indeed let us denote by $A$ the set of exponents $(i, j)$ such that $\bar{p}_{i, j} \neq 0$. We call it the support of $\sigma^{h}$. By a change of variable $t_{i}=\frac{u_{i}}{1+u_{i}}$, the polynomials $B_{m_{1}}^{i}\left(t_{1}\right) B_{m_{2}}^{j}\left(t_{2}\right)$ are transformed into $\left(\begin{array}{c}m_{1} \\ i\end{array}\right)\left(\begin{array}{c}m_{2} \\ j\end{array}\right) u_{1}^{i} u_{2}^{j}$ up to the factor $\delta\left(u_{1}, u_{2}\right)=\frac{1}{\left(1+u_{1}\right)^{m_{1}}\left(1+u_{2}\right)^{m_{2}}}$. After this change of variables, each coordinates $\sigma_{i}\left(u_{1}, u_{2}\right)$ is up to the denominator $\delta\left(u_{1}, u_{2}\right)$, a 
polynomial with support in $A$. The toric variety behind this construction is thus the toric variety $\mathcal{T}_{A}$ parameterized by the set of monomials $u_{1}^{i} u_{2}^{j}$ with $(i, j) \in$ $A$. Using the projective coordinates of $\mathcal{T}_{A}$ [Cox95], another description of these pacthes, more adapted to numerical computation can be given (see [?, ?] for more details). Le us assume here that $A \subset \mathbb{Z}^{2}$ is an integer polytope, described by the inequalities $h_{l}(m) \geq 0, l=1, \ldots, s$ iff $m \in A$. The toric patch of $A$ with control points $\bar{p}_{m}$ with $m \in A$ is by definition the image of $A_{\mathbb{R}}:=A \otimes \mathbb{R} \subset \mathbb{R}^{2}$ by the projective map

$$
\tau_{A}\left(t_{1}, t_{2}\right) \in A_{\mathbb{R}} \mapsto \sum_{m \in A} \bar{p}_{m} c_{m} \prod_{l=1}^{s} h_{l}\left(t_{1}, t_{2}\right)^{h_{l}(m)}
$$

for some binomial-type coefficients $c_{m} \in \mathbb{Q}$. The is map transform $A_{\mathbb{R}}$ into a surface with the same shape.Indeed, some properties of the polytope $A$ prescibed the geometry of $\tau_{A}$.

The toric resultant theory described in section 3.3 applies directly in this case. By BKK theorem [Ber75], the degree of the toric patch $\sigma_{\mathcal{T}_{A}}$ is twice the volume of $A$ for generic values of the control points $\bar{p}_{m}$. Its implicit equation is the toric resultant in $u_{1}, u_{2}$ of the numerators of

$$
\sigma_{1}\left(u_{1}, u_{2}\right)-x_{1} \sigma_{0}\left(u_{1}, u_{2}\right), \sigma_{2}\left(u_{1}, u_{2}\right)-x_{2} \sigma_{0}\left(u_{1}, u_{2}\right), \sigma_{3}\left(u_{1}, u_{2}\right)-x_{3} \sigma_{0}\left(u_{1}, u_{2}\right) .
$$

EXAmple 4.13. Consider a Pillow patch with support in $A=\{(1,0),(0,1),(1,1),(2,1),(1,2)\}$ :

$$
\begin{aligned}
& x_{0}=-\frac{1}{2} F_{1,0}(\mathbf{t})-\frac{1}{2} F_{0,1}(\mathbf{t})-\frac{1}{2} F_{1,1}(\mathbf{t})-\frac{1}{2} F_{2,1}(\mathbf{t})-\frac{3}{2} F_{1,2}(\mathbf{t}), \\
& x_{1}=-\frac{1}{2} F_{1,0}(\mathbf{t})-\frac{1}{2} F_{0,1}(\mathbf{t})-\frac{1}{2} F_{1,1}(\mathbf{t})-\frac{1}{2} F_{2,1}(\mathbf{t})-\frac{3}{2} F_{1,2}(\mathbf{t}), \\
& x_{2}=-\frac{1}{2} F_{1,0}(\mathbf{t})+\frac{3}{2} F_{0,1}(\mathbf{t})-\frac{1}{2} F_{1,1}(\mathbf{t})+F_{1,2}(\mathbf{t})+\frac{7}{F} 1,2(\mathbf{t}), \\
& x_{3}=2 F_{1,0}(\mathbf{t}) F_{0,1}(\mathbf{t})+\frac{7}{4} F_{1,1}(\mathbf{t})-3 F_{2,1}(\mathbf{t})-4 F_{1,2}(\mathbf{t})
\end{aligned}
$$

where $F_{i, j}(\mathbf{t})=B_{2}^{i}\left(t_{1}\right) B_{2}^{j}\left(t_{2}\right)$. Using the command spresultant, we obtain yields a $18 \times 18$ matrix, whose determinant of degree 6 factors as the toric resultant of degree 4 and a product of parasite linear factors:

$$
\begin{aligned}
& 200\left(2 x_{2}+x_{3}+2\right)^{2}\left(-247730 x_{2}{ }^{2} x_{3} x_{1}+114400 x_{1}{ }^{4}-1823194 x_{1}+1347025 x_{2}\right. \\
& +61332 x_{2}{ }^{3} x_{3}-17956 x_{3}{ }^{3} x_{1}-328050 x_{2} x_{1}{ }^{3}+186323 x_{2} x_{3}{ }^{2}+28900 x_{3}{ }^{3}-151240 x_{3} x_{1}{ }^{3} \\
& +77684 x_{3}{ }^{2} x_{1}{ }^{2}-2518405 x_{2} x_{1}+624498 x_{3}+1554 x_{3}{ }^{4}-115575 x_{2} x_{3}{ }^{2} x_{1}+728615 \\
& -176855 x_{2}{ }^{3} x_{1}+285871 x_{2}{ }^{3}-1166938 x_{3} x_{1}+334100 x_{2} x_{1}{ }^{2} x_{3}+1714989 x_{1}{ }^{2} \\
& -250397 x_{3}{ }^{2} x_{1}+201416 x_{3}{ }^{2}+866788 x_{2} x_{3}-720510 x_{1}{ }^{3}+726892 x_{1}{ }^{2} x_{3}+42957 x_{2}{ }^{2} x_{3}{ }^{2} \\
& +13352 x_{2} x_{3}{ }^{3}+1571470 x_{2} x_{1}{ }^{2}-1076780 x_{2} x_{1} x_{3}+359365 x_{2}{ }^{2} x_{1}{ }^{2}-1157370 x_{2}{ }^{2} x_{1} \\
& \left.+931973 x_{2}{ }^{2}+399958 x_{2}{ }^{2} x_{3}+32788 x_{2}{ }^{4}\right)
\end{aligned}
$$

\section{Algorithms for algebraic CAGD problems}

In this section, we illustrate the use of resultant techniques for specific problems occurring in Computer Aided Geometric Design. Our main point is here to produce methods which will apply for a class of systems. Once the geometry of these systems is analyzed, one is able to precompute the solution of the problem by using an adapted resultant formulation and to reduce the numerical solving part to linear algebra computation. This is what we are going to show now on some examples.

5.1. Computing the inverse image of a point on the surface. The resultant is by nature a projection tool. Keeping the way it has been constructed, yields a mean to recover of the fiber of this projection.

Given a point $\mathbf{y}=\left(y_{0}, y_{1}, y_{2}\right)$ on a parameterized surface (4), we want for instance to compute the parameter(s) $\mu=\left(\mu_{1}, \mu_{2}\right)$ such that $\sigma(\mu)=\mathbf{y}$. 
Using one of the resultant formulations of section 4, we obtain a square matrix

$$
\left.\mathrm{R}(\mathbf{x})=\left(S_{i, j}(\mathbf{x})\right)\right\} \mathbf{p}(\mathbf{t})
$$

whose determinant is a multiple of the implicit equation of $\sigma$. This matrix is obtained by decomposing polynomials which vanishes when $\mathbf{x}=\sigma(\mathbf{t})$, into a set of polynomials (usually monomials) $\mathbf{p}(\mathbf{t})=\left\{p_{1}(\mathbf{t}), \ldots, p_{s}(\mathbf{t})\right\}\left(\mathrm{R}\left(\sigma_{\mathbf{t}}\right)^{t} \mathbf{p}(\mathbf{t})=0\right)$. In most of the cases, the set $\mathbf{p}$ of polynomials contains $1, t_{1}, t_{2}$.

By construction, substituting $\mathbf{x}$ by the value $\mathbf{y}$ yields a matrix $R(\mathbf{y})$ whose determinant vanishes. Moreover, we have $\mathrm{R}(\mathbf{y})^{t} \mathbf{p}(\mu)=0$ since the columns of $R(\mathbf{x})$ represent polynomials which vanishes when $\mathbf{x}=\sigma(\mathbf{t})$. This gives us a way to recover the inverse image of $\mathbf{y}$. Two cases have to be considered:

(1) The kernel $\operatorname{Ker}\left(\mathrm{R}^{t}(\mathbf{y})\right)$ is of dimension 1.

(2) The kernel of $\mathrm{R}^{t}(\mathbf{y})$ is of higher dimension.

In the first case, since $x_{0}=\sigma(\mu)$ the kernel is generated by $\mathbf{p}(\mu)$. Assuming that $\mathbf{p}(t)=\left[1, t_{1}, t_{2}, \ldots\right]$ and that $W=\left[w_{1}, w_{t_{1}}, w_{t_{2}}, \ldots\right]$ generates $\operatorname{Ker}\left(S^{t}(\mathbf{y})\right)$, we deduce the coordinates of the unique inverse image

$$
t_{1}=\frac{w_{t_{1}}}{w_{1}}, t_{2}=\frac{w_{t_{2}}}{w_{1}} .
$$

In the second case, we denote by $W_{1}, \ldots, W_{k}$ a basis of $\operatorname{Ker}\left(S^{t}(\mathbf{y})\right)$ whose coordinates are indexed by the polynomials $\mathbf{p}(\mathbf{t})$. These vectors represent linear forms $\Lambda_{1}, \ldots, \Lambda_{k}$ which vanish on the polynomials represented by the columns of $\mathrm{R}(\mathbf{x})$.

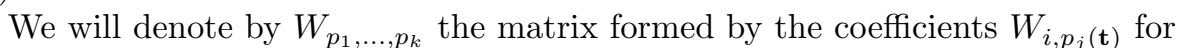
$p_{j}(\mathbf{t}) \in \mathbf{p}(\mathbf{t})$ and $1 \leq i, j \leq k$. Assume that there exists a subvector $\mathbf{b}_{0}(\mathbf{t})$ of $\mathbf{p}(\mathbf{t})$ such that $W_{\mathbf{b}_{0}(\mathbf{t})}$ is invertible and $t_{1} \mathbf{b}_{0}(\mathbf{t})$ and $t_{2} \mathbf{b}_{0}(\mathbf{t})$ are subvectors of $\mathbf{p}(\mathbf{t})$, then any inverse image $\mu=\left(\mu_{1}, \mu_{2}\right)$ of $\mathbf{y}, B(\mu)$ is an element of the $\operatorname{kernel} \operatorname{Ker}\left(S^{t}(\mathbf{y})\right)$, so that

$$
B(\mu)=W W_{\mathbf{b}_{0}}^{-1} \mathbf{b}_{0}(\mu)
$$

In particular, denoting $\mathbf{b}_{1}=t_{1} \mathbf{b}_{0}(\mathbf{t}), \mathbf{b}_{2}=t_{2} \mathbf{b}_{0}(\mathbf{t})$, we have

$$
\mathbf{b}_{1}(\mu)=W_{\mathbf{b}_{1}} W_{\mathbf{b}_{0}}^{-1} \mathbf{b}_{0}(\mu)=\mu_{1} \mathbf{b}_{0}(\mu)
$$

so that $\mathbf{w}=W_{\mathbf{b}_{0}}^{-1} \mathbf{b}_{0}(\mu)$ satisfies

$$
\left(W_{\mathbf{b}_{1}}-\mu_{1} W_{\mathbf{b}_{0}}\right) \mathbf{w}=0 .
$$

Similarly we have $\left(W_{\mathbf{b}_{2}}-\mu_{2} W_{\mathbf{b}_{0}}\right) \mathbf{w}=0$.

Therefore computing the set of generalized eigenvalues $\left(\mu_{1}, \mu_{2}\right)$ satisfying

$$
\left(W_{\mathbf{b}_{1}(\mathbf{t})}-t_{1} W_{\mathbf{b}_{0}(\mathbf{t})}\right) \mathbf{w}=0,\left(W_{\mathbf{b}_{2}(\mathbf{t})}-t_{2} W_{\mathbf{b}_{0}(\mathbf{t})}\right) \mathbf{w}=0
$$

for some vector $\mathbf{w} \neq 0$ contains the coordinates of the inverse images of $\mathbf{y}$.

EXAMPLE 5.1. Let us illustrate this construction on the case of a Steiner surface parameterized by

$$
\sigma: \mathbf{t}=\left(t_{1}: t_{2}\right) \mapsto\left(q_{0}(\mathbf{t}): q_{1}(\mathbf{t}): q_{2}(\mathbf{t}): q_{3}(\mathbf{t})\right)
$$

where the $q_{i}$ are of degree 2, with no base points. See [SA85], [AS01].

Using the construction 3.1, we obtain a $6 \times 6$ matrix $\mathrm{R}(\mathbf{x})$ of coefficients of polynomials

$$
\left[q_{1}(\mathbf{t})-x_{1} q_{0}(\mathbf{t}), q_{2}(\mathbf{t})-x_{2} q_{0}(\mathbf{t}), q_{y}(\mathbf{t})-x_{3} q_{0}(\mathbf{t}), \tau_{1}(\mathbf{t}, \mathbf{x}), \tau_{2}(\mathbf{t}, \mathbf{x}), \tau_{3}(\mathbf{t}, \mathbf{x})\right] .
$$


The columns of $\mathrm{R}(\mathbf{x})$ are indexed by the monomials $1, t_{1}, t_{2}, t_{1}^{2}, t_{1} t_{2}, t_{2}^{2}$.

The determinant of $\mathrm{R}(\mathbf{x})$ is the implicit equations of the Steiner surface, of degree 4. The dimension of the kernel $\operatorname{ker}(\mathrm{R}(\mathbf{y}))$ is the number of inverse image of $\mathbf{y}$. It is at most 3 .

When this dimension is 1 , we deduce the coordinates of the inverse image as the solution of $W_{t_{1}}-u_{1} W_{1}=0, W_{t_{2}}-u_{2} W_{1}=0$.

When the dimension is 2 , the point $\mathbf{y}$ is on one of the double line of the surface. The two inverse images are obtained either from the generalized eigenvalue of $\left(W_{t_{1}, t_{1}^{2}}-u_{1} W_{1, t_{1}}\right) \mathbf{w}=0,\left(W_{t_{2}, t_{1} t_{2}}-u_{2} W_{1, t_{1}}\right) \mathbf{w}=0$, or $\left(W_{t_{1} t_{2}, t_{2}^{2}}-u_{1} W_{1, t_{2}}\right) \mathbf{w}=$ $0,\left(W_{t_{22}, t_{1} t_{2}}-u_{2} W_{1, t_{2}}\right) \mathbf{w}=0$.

When the dimension is 3 , the point $\mathbf{y}$ is the triple point of the surface. Its 3 inverse images can be computed from generalized eigenvalue problems $\left(W_{t_{1}, t_{1}^{2}, t_{1} t_{2}}-\right.$ $\left.u_{1} W_{1, t_{1}, t_{2}}\right) \mathbf{w}=0,\left(W_{t_{2}, t_{1} t_{2}, t_{2}^{2}}-u_{2} W_{1, t_{1}, t_{2}}\right) \mathbf{w}$.

5.2. Intersection of parameterized curves and surfaces. Computing the implicit equation of a parametric rational surface can be used in some problems in CAGD such as intersecting of two parametric surfaces. One way to do this is to find the implicit equation of one surface and to replace the other in this equation. Now we will see how to intersect a parametric curve and an implicit surface using resultants.

Let $g_{1}, g_{2}, g_{3}, d_{1}, d_{2}, d_{3}$ be polynomials in one variable $s$ and let

$$
C:\left\{\begin{array}{l}
x_{1}=\frac{g_{1}(s)}{d_{1}(s)} \\
x_{2}=\frac{g_{2}(s)}{d_{2}(s)} \\
x_{3}=\frac{g_{3}(s)}{d_{3}(s)}
\end{array}\right.
$$

be the rational parametric curve they define and $S=\left\{(a, b, c) \in \mathbb{K}^{3}: g(a, b, c)=0\right\}$ be an implicit surface. Our goal is to compute the intersection of $C$ and $S$ by means of resultant. We consider the polynomial system

$$
g\left(x_{1}, x_{2}, x_{3}\right)=x_{1} d_{1}(s)-g_{1}(s)=x_{2} d_{2}(s)-g_{2}(s)=x_{3} d_{3}(s)-g_{3}(s)=0
$$

and we compute the resultant matrix $\mathrm{R}(s)=R_{d} s^{d}+\cdots+R_{0}$ by hiding the variable $s$ (i.e. these polynomials are viewed as elements in $\left.(\mathbb{K}[s])\left[x_{1}, x_{2}, x_{3}\right]\right)$. The coefficients $R_{i}$ are numerical matrices of the same size than $\mathrm{R}(s)$ and the degree $d$ is the maximum of degrees of polynomials $d_{i}$ and $g_{i}$.

We are looking for the values of $s$ such that this system has a solution $\left(x_{1}, x_{2}, x_{3}\right)$. That is, a vector $v=v\left(x_{1}, x_{2}, x_{3}\right)$ indexed by the rows of $\mathrm{R}(s)$ such that $R(s)^{t} v=0$.

If the matrix $R_{d}$ is invertible this is equivalent to $s^{d} v+s^{d-1}\left(R_{d}^{-1} R_{d-1}\right)^{t} v+$ $\cdots+\left(R_{d}^{-1} R_{0}\right)^{t} v=0$, that is the vector $w={ }^{t}\left(v, s v, \ldots, s^{d-1} v\right)$ is an eigenvector of the matrix

$$
\left(\begin{array}{cccc}
0 & \mathbb{I} & & \\
& \ddots & \ddots & \\
& & 0 & \mathbb{I} \\
-\left(R_{d}^{-1} R_{0}\right)^{t} & \ldots & & -\left(R_{d}^{-1} R_{d-1}\right)^{t}
\end{array}\right)
$$


where $\mathbb{I}$ is the identity matrix. If $R_{d}$ is not invertible, $w$ is a solution of the generalized eigenvector problem

$$
\left[\left(\begin{array}{cccc}
0 & \mathbb{I} & & \\
& \ddots & \ddots & \\
& & 0 & \mathbb{I} \\
-R_{0}^{t} & \cdots & & R_{d-1}^{t}
\end{array}\right)-\left(\begin{array}{cccc}
\mathbb{I} & & & \\
& \ddots & & \\
& & \mathbb{I} & \\
& & & R_{d}^{t}
\end{array}\right)\right]\left(\begin{array}{c}
v \\
s v \\
\vdots \\
s^{d-1} v
\end{array}\right)=0 .
$$

EXAMPLE 5.2.

Such tool can be useful in ray tracing techniques which involve the intersection of a surface with a line. Similarly computing a bounding volume of a parameterized surface can be deduced from the intersection of surface and its dual (the set of of vectors $\partial_{t_{1}} \sigma \wedge \partial_{t_{2}} \sigma$ also parameterized by $\left.\left(t_{1}, t_{2}\right)\right)$ with lines.

5.3. Detection of singularities. Another important problem in computer aided design is the detection of singularities of a $3 D$-surface.

Let $S=\left\{(a, b, c) \in \mathbb{K}^{3}: f(a, b, c)=0\right\}$ be a surface given by a polynomial $f \in \mathbb{K}[x, y, z]$.

A point $(a, b, c)$ in $S$ is singular if $\frac{\partial f}{\partial x_{1}}(a, b, c)=\frac{\partial f}{\partial x_{2}}(a, b, c)=\frac{\partial f}{\partial x_{3}}(a, b, c)=0$.

It is clear that the surface $S$ has singular points if and only if the resultant of the polynomials $f, \frac{\partial f}{\partial x_{1}}, \frac{\partial f}{\partial x_{2}}, \frac{\partial f}{\partial x_{3}}$ is equal to 0 .

EXAMPLE 5.3. Let $f=x_{1}\left(x_{1}^{2}-x_{2}^{2}\right)+x_{3}^{2}\left(1+x_{3}\right)+\frac{2}{5} x_{1} x_{2}+\frac{2}{5} x_{2} x_{3}$. The partial derivatives of this cubic are

$\frac{\partial f}{\partial x_{1}}=3 x_{1}^{2}-x_{2}^{2}+\frac{2}{5} x_{2}, \frac{\partial f}{\partial x_{2}}=-2 x_{1} x_{2}+\frac{2}{5} x_{1}+\frac{2}{5} x_{3}, \frac{\partial f}{\partial x_{3}}=2 x_{3}+3 x_{3}^{2}+\frac{2}{5} x_{2}$.

The resultant of $f, \frac{\partial f}{\partial x_{1}}, \frac{\partial f}{\partial x_{2}}, \frac{\partial f}{\partial x_{3}}$ vanishes, then this cubic has singularities.

In the case, where the surface $S$ is given in a parametric form, singularities or self-intersection points can also be recovered under some hypotheses. As in section, using one of the adapted resultant formulation of section 4, we obtain a $N \times N$ matrix $\mathrm{R}(\mathbf{t})$ whose columns are indexed by polynomials $\mathbf{p}(\mathbf{t})=\left\{p_{1}(\mathbf{t}), \ldots, p_{s}(\mathbf{t})\right\}$ such that $\mathrm{R}(\sigma(\mathbf{t}))^{t} \mathbf{p}(\mathbf{t})=0$. Its determinant is a multiple of the implicit equation of $S$. We assume here that $p(\mathbf{t})$ is of the form $\mathbf{p}(t)=\left[1, t_{1}, t_{2}, \ldots\right]$ and that for generic point $\mathbf{x} \in S, \mathrm{R}(\mathbf{x})$ is of rank $N-1$. Consider now a multiple point $\mathbf{y}$ of $S$ such that there exists $\mu \neq \mu^{\prime} \in \mathbb{K}^{2}$, such that $\mathbf{y}=\sigma(\mu)=\sigma\left(\mu^{\prime}\right)$. Then $\mathbf{p}(\mu)$ and $\mathbf{p}\left(\mu^{\prime}\right)$ are two independent vectors in $\operatorname{Ker}\left(\mathrm{R}(\mathbf{y})^{t}\right)$. Similarly, if $\mu$ is a singular point of the parameterization of $S$, we get at least two independent vectors $\mathbf{p}(\mu)$ and $\partial \mathbf{p}(\mu)$ in $\operatorname{Ker}\left(\mathrm{R}(\mathbf{y})^{t}\right)$. Thus a necessary condition for $\mathbf{y}$ to be a singular point of $S$ is that $\mathrm{R}(\mathbf{y})$ is of rank $\leq N-2$. In other words, the auto-intersection points or the singular points of the parameterized surface $S$ are located on the zero-set of all $(N-1) \times(N-1)$ minors of $\mathrm{R}(\mathbf{x})$.

\section{References}

[AS01] F. Ariès and R. Senoussi. An implicitization algorithm for rational surfaces with no base points. J. of Symbolic Computation, 31:357-365, 2001.

[B6́4] E. Bézout. Recherches sur les degrés des équations résultantes de l'évanouissement des inconnues et sur les moyens qu'il convient d'employer pour trouver ces équations. Hist de l'Aca. Roy. des Sciences, pages 288-338, 1764. 
[BCD02] Laurent Busé, David Cox, and Carlos D'Andrea. Implicitization of surfaces in $\mathbb{P}^{3}$ in the presence of base points. math.AG/0205251, Preprint, 2002.

[BEM00] L. Busé, M. Elkadi, and B. Mourrain. Generalized resultant over unirational algebraic varieties. J. of Symbolic Computation, 29:515-526, 2000.

[BEM01] L. Busé, M. Elkadi, and B. Mourrain. Residual resultant of complete intersection. $J$. of Pure and Applied Algebra, 164:35-57, 2001.

[Ber75] D.N. Bernstein. The number of roots of a system of equations. Funct. Anal. and Appl., 9(2):183-185, 1975.

[BJ02] Laurent Busé and Jean-Pierre Jouanolou. On the closed image of a rational map and the implicitization problem. math.AG/0210096, Preprint, 2002.

[BKM90] W. Bruns, A. R. Kustin, and M. Miller. The resolution of the generic residual intersection of a complete intersection. Journal of Algebra, 128:214-239, 1990.

[Bus01a] Laurent Busé. Étude du résultant sur une variété algébrique. PhD thesis, Université de Nice Sophia-Antipolis, 2001.

[Bus01b] Laurent Busé. Residual resultant over the projective plane and the implicitization problem. In B. Mourrain, editor, Proc. Annual ACM Intern. Symp. on Symbolic and Algebraic Computation, pages 48-55, London, Ontario, 2001. New-York, ACM Press.

[CE93] J. Canny and I. Emiris. An efficient algorithm for the sparse mixed resultant. In G. Cohen, T. Mora, and O. Moreno, editors, Proc. Intern. Symp. on Applied Algebra, Algebraic Algorithms and Error-Corr. Codes (Puerto Rico), volume 673 of Lect. Notes in Comp. Science, pages 89-104. Springer, 1993.

[CGZ00] David A. Cox, Ronald Goldman, and Ming Zhang. On the validity of implicitization by moving quadrics for rationnal surfaces with no base points. J. Symbolic Computation, 29,2000

[Cha93] M. Chardin. The resultant via a Koszul complex. In F. Eyssette and A. Galligo, editors, Computational Algebraic Geometry, volume 109 of Prog. in Mathematics, pages 29-39. Birkhäuser, Boston, 1993. (Proc. MEGA '92, Nice).

[CLO97] D. Cox, J. Little, and D. O'Shea. Using Algebraic Geometry. Springer-Verlag, New York, 1997.

[Cox95] D. Cox. The homogeneous coordinate ring of a toric variety. J. Algebraic Geom., 4(1):17$50,1995$.

[CP93] J. Canny and P. Pedersen. An algorithm for the Newton resultant. Technical Report 1394, Comp. Science Dept., Cornell University, 1993.

[CU00] M. Chardin and B. Ulrich. Liaison and Castelnuovo-Mumford regularity. Preprint, 2000.

[D'A01] Carlos D'Andréa. Resultants and moving surfaces. J. of Symbolic Computation, 31, 2001.

[D'A02] Carlos D'Andréa. Macaulay style formulas for sparse resultants. Trans. Amer. Math. Soc., 354:2595-2629, 2002.

[DD01] C. D'Andrea and A. Dickenstein. Generalized Macaulay formulas for the multivariate resultant. Jour. Pure and Applied Algebra, 2001.

[Dem84] M. Demazure. Une définition constructive du résultant. Notes Informelles de Calcul Formel, Centre de Mathématiques de l'Ecole Polytechnique, May 1984.

[Eis94] D. Eisenbud. Commutative Algebra with a view toward Algebraic Geometry, volume 150 of Graduate Texts in Math. Berlin, Springer-Verlag, 1994.

[EM02] M Elkadi and B. Mourrain. Residue and implicitization problem for rational surfaces. Preprint, 2002.

[GH78] Ph. Griffiths and J. Harris. Principles of Algebraic Geometry. Wiley Interscience, New York, 1978.

[GKZ94] I.M. Gelfand, M.M. Kapranov, and A.V. Zelevinsky. Discriminants, Resultants and Multidimensional Determinants. Boston, Birkhäuser, 1994.

[GV97] L. Gonzalez-Vega. Implicitization of parametric curves and surfaces by using multidimensional newton formulae. J. Symb. Comput., 23(2-3):137-151, 1997.

[Har77] R. Hartshorne. Algebraic Geometry. Springer-Verlag, 1977.

[Jou91] J.P. Jouanolou. Le formalisme du résultant. Adv. in Math., 90(2):117-263, 1991.

[Jou96] J.-P. Jouanolou. Résultant anisotrope: Compléments et applications. The electronic journal of combinatorics, 3(2), 1996.

[Jou97] J.P. Jouanolou. Formes d'inertie et résultant: un formulaire. Adv. in Math., 126(2):119250, 1997.

[Khe02] Amit Khetan. The resultant of an unmixed bivariate system. Preprint, math.AG/0209115, 2002. 
[KSZ92] M. M. Kapranov, B. Sturmfels, and A.V. Zelevinsky. Chow polytopes and general resultants. Duke Math. Journal, 67:189-218, 1992.

[Mac02] F.S. Macaulay. Some formulae in elimination. Proc. London Math. Soc., 1(33):3-27, 1902 .

[SA85] T.W. Sederberg and D.C. Anderson. Steiner surface patches. IEEE Comput. Graphics Appl., 5(5):23-36, 1985.

[SC95] T.W. Sederberg and F Chen. Implicitization using moving curves and surfaces. Proceedings of SIGGRAPH, pages 120-160, 1995.

[vdW50] B.L. van der Waerden. Modern Algebra. F. Ungar Publishing Co., New York, 3rd edition, 1950.

[WZ94] Jerzy Weyman and Andrei Zelevinsky. Determinantal formulas for multigraded resultants. J. of Algebraic Geometry, 3:569-597, 1994.

\section{Appendix A. Determinants of four-term exact complexes of vector spaces}

We expose briefly a known method (going back to Cayley) for computing determinants of complexes in the simple case of interest in this paper of four-term exact complexes of finite-dimensional vector spaces. For a complete treatment of the subject we refer to [GKZ94], appendix A.

Suppose that we have a four-term exact complex of vector spaces

$$
0 \rightarrow V_{3} \stackrel{\partial_{2}}{\longrightarrow} V_{2} \stackrel{\partial_{1}}{\longrightarrow} V_{1} \stackrel{\partial_{0}}{\longrightarrow} V_{0} \rightarrow 0 \text {. }
$$

Since $\partial_{0}$ is surjective, $V_{1}$ decomposes into $V_{0} \oplus V_{1}^{\prime}$ and $\partial_{1}=\left(\begin{array}{cc}\phi_{0} & m_{0}\end{array}\right)$ with $\operatorname{det}\left(\phi_{0}\right) \neq 0$. Now, since $\operatorname{Im}\left(\partial_{1}\right)=\operatorname{ker}\left(\partial_{0}\right), V_{2}$ decomposes into $V_{1}^{\prime} \oplus V_{2}$ and $\partial_{1}=$ $\left(\begin{array}{cc}m_{1} & m_{2} \\ \phi_{1} & m_{3}\end{array}\right)$ with $\operatorname{det}\left(\phi_{1}\right) \neq 0$. Finally since $\partial_{2}$ is injective and $\operatorname{Im}\left(\partial_{2}\right)=\operatorname{ker}\left(\partial_{1}\right)$, we have $\partial_{2}=\left(\begin{array}{c}m_{4} \\ \phi_{2}\end{array}\right)$ with $\operatorname{det}\left(\phi_{2}\right) \neq 0$. The determinant of the complex $(7)$ is then obtained as the quotient $\frac{\operatorname{det}\left(\phi_{0}\right) \operatorname{det}\left(\phi_{2}\right)}{\operatorname{det}\left(\phi_{1}\right)}$, and is independent of its construction.

Note that if $V_{3}=0$, we can make the same decomposition which shows that the determinant of $(7)$ is a quotient of the form $\frac{\operatorname{det}\left(\phi_{0}\right)}{\operatorname{det}\left(\phi_{1}\right)}$. Similarly, when moreover $V_{2}=0$, we recover the standard notion of determinant since the determinant of (7) is then the determinant of the map $\partial_{0}$.

Laurent Busé, UNSA, UMR 6621, Parc Valrose, B.P. 71, 06108 Nice, France

E-mail address: lbuse@math.unice.fr

Mohamed Elkadi, UnSA, UMR 6621, Parc Valrose, B.P. 71, 06108 Nice, France

E-mail address: elkadi@math.unice.fr

Bernard Mourrain, inRIA, GALAAD, B.P. 93, 06902 Sophia Antipolis, France

E-mail address: mourrain@sophia.inria.fr 